Supporting Information for

\title{
Dual Functional Pd-Catalyzed Multicomponent Reaction by Umpolung Chemistry of the Oxygen Atom in Electrophiles
}

Wangyujing Han, $\ddagger$ Jie Yu, $\$$ Zhenghui Kang, Longlong Song, Rou Pi, Suzhen Dong, Yuqing Xiong, Fei Xia, Zi Li, and Shunying Liu*+

Shanghai Engineering Research Center of Molecular Therapeutics and New Drug Development, School of Chemistry and Chemical Engineering, East China Normal University, 3663 North Zhongshan Road, Shanghai, China 200062

Email: syliu@sist.ecnu.edu.cn.

\section{Table of Contents}

1. Optimization of Reaction Conditions.....................................S2

2. Control experiments ..................................................... 2

3. LC-MS spectra of reaction solution and 5a..........................................S3

4. UV-vis Spectra of the mixture of the $[\mathrm{Pd}(\text { allyl }) \mathrm{Cl}]_{2}$ and $\mathrm{PhNO}$ in DMSO_........S4

5. Operando IR spectroscopy specie lines of the $[\mathrm{Pd}(\text { allyl }) \mathrm{Cl}]_{2}$ and nitrosobenzene in

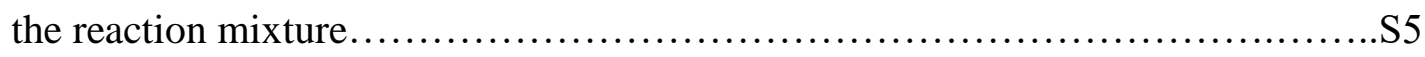

6. ${ }^{1} \mathrm{H}$ NMR of the mixture of the $[\mathrm{Pd}(\text { allyl }) \mathrm{Cl}]_{2}$ and nitrosobenzene in DMSO....S6-S7

7. X-ray Diffraction Parameters and Data of 4 p................................ 89

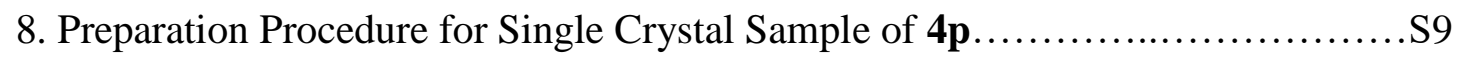

9. NMR Spectra of Compounds.............................................S10-S27 


\section{Optimization of Reaction Conditions}

Table S1 Optimization of Reaction Conditions

a) ${ }^{a}$<smiles>COC(OC)C(=N)c1ccccc1</smiles>

b)

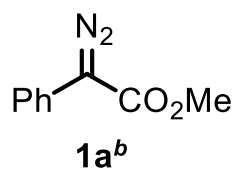

$[\mathrm{Pd}(\text { allyl }) \mathrm{Cl}]_{2}$ $1 \mathrm{~mol} \%$ $\underset{\mathrm{DCM}, 25^{\circ} \mathrm{C}}{\stackrel{4 \AA \mathrm{MS}}{\mathbf{2}} \mathbf{a}, \mathbf{a}} \quad \mathbf{4 a} \quad$ yield: $40 \%$

$[\mathrm{Pd}(\text { allyl }) \mathrm{Cl}]_{2}$ $0.5-10 \mathrm{~mol} \%$ $\underset{\mathrm{DCM}, 25^{\circ} \mathrm{C}}{\stackrel{4 \AA \mathrm{MS}, \mathbf{2 a}, \mathbf{3 a}}{\longrightarrow}} \quad \mathbf{4 a} \quad$ yield: $75 \%$

${ }^{a}$ Reaction conditions: 1a:2a:3a=1:1:1, $0.05 \mathrm{mmol}$ scale of $\mathbf{3 a}$.

${ }^{b}$ Added into the reaction solution via a spring pump

\section{Control experiments ${ }^{a}$}

a)<smiles>COC(=O)C(=N)c1ccccc1</smiles>

1a

b)<smiles>COC(OC)C(=N)c1ccccc1</smiles>

c)

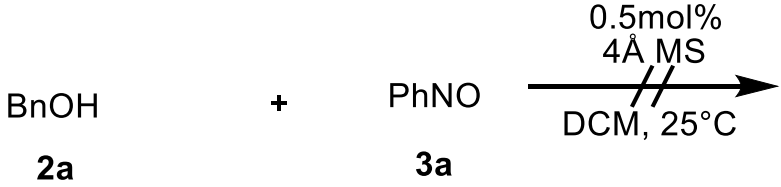

5a yield: $<5 \%$

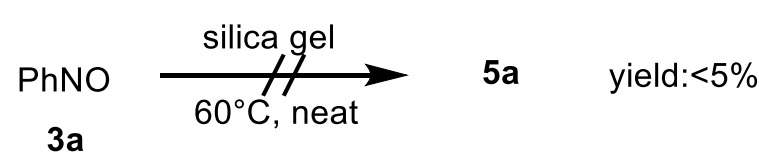

no reaction

\footnotetext{
${ }^{a} \mathbf{1} \mathbf{a}$ was added into the reaction solution via a spring pump
}

Scheme S1 Control experiments 
3. LC-MS spectra of reaction solution and 5a
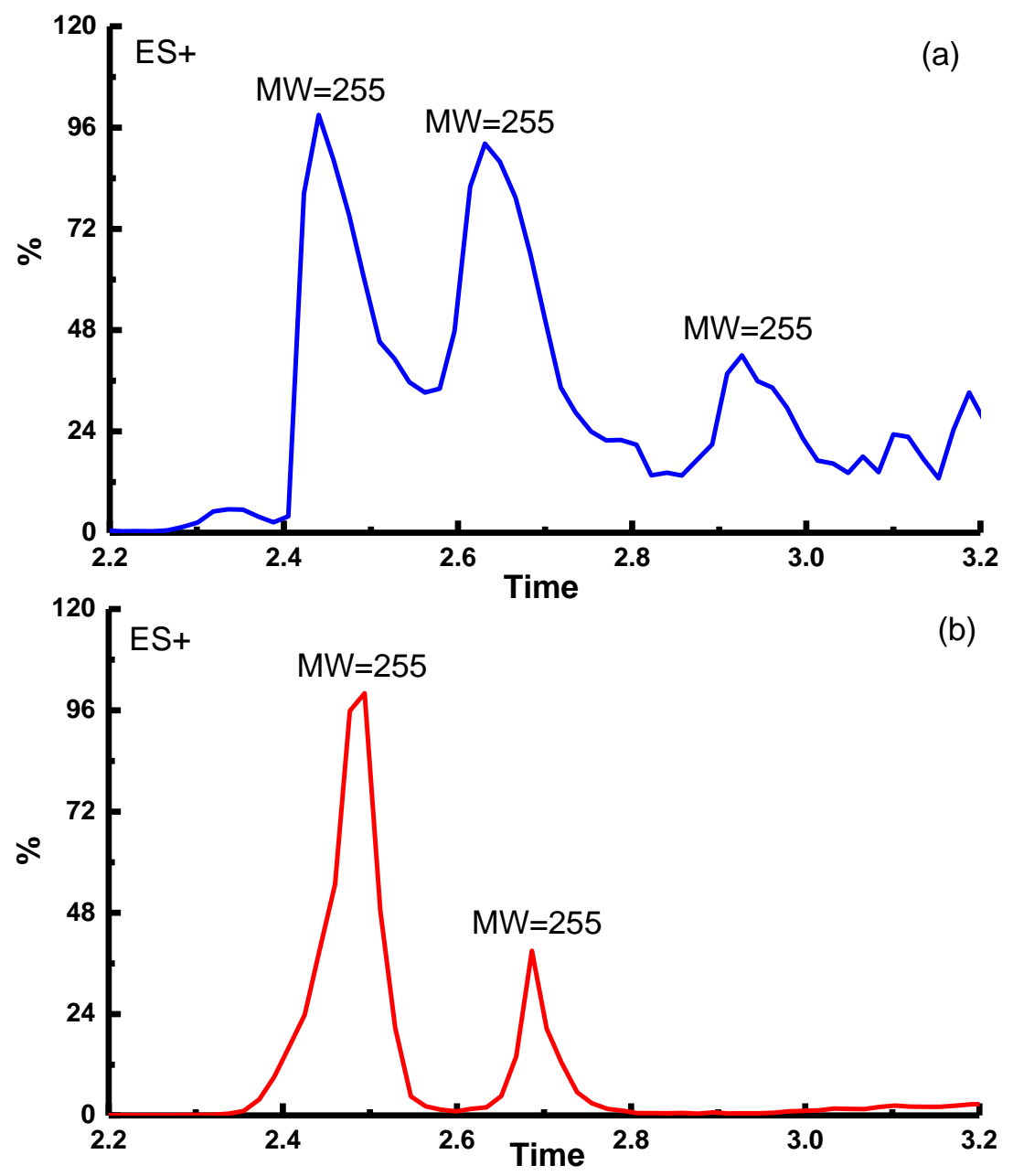

Figure S1 Total Ion Chromatography (TIC) spectra of reaction solution and 5a by the time-of-flight mass chromatogram of the positive ion. 


\section{UV-vis Spectra of the mixture of the $[\mathrm{Pd}(\text { allyl }) \mathrm{Cl}]_{2}$ and $\mathrm{PhNO}$ in}

\section{DMSO}

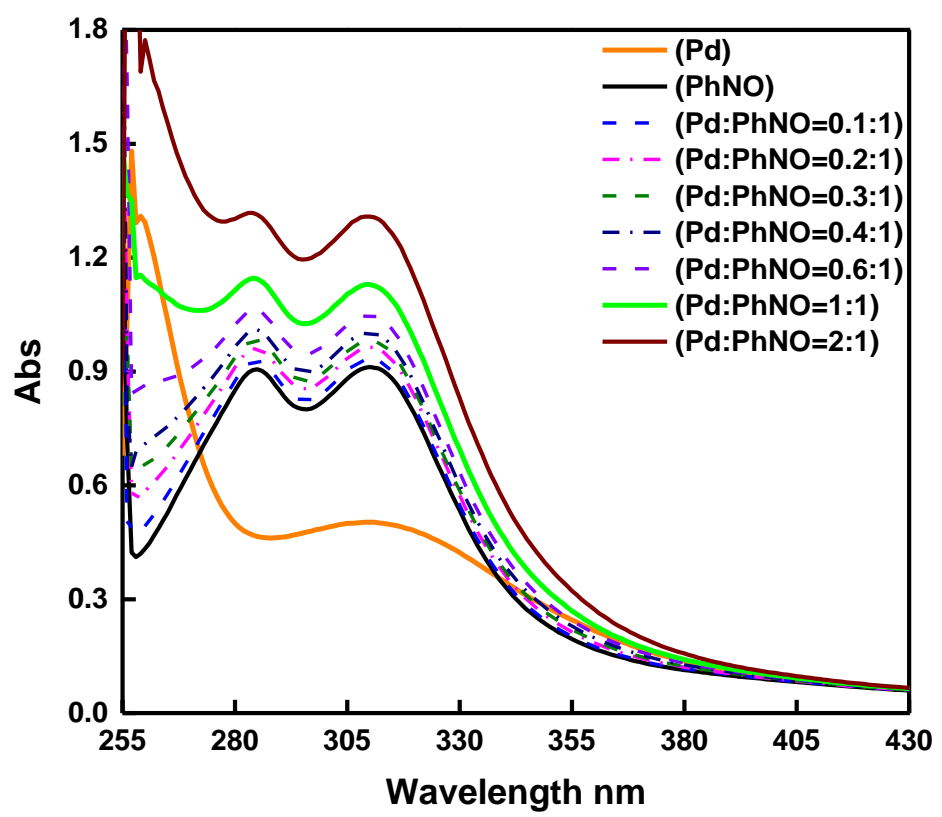

Figure S2. UV-vis Spectra of the mixture of the $[\mathrm{Pd}(\text { allyl }) \mathrm{Cl}]_{2}$ and $\mathrm{PhNO}$ in DMSO with the ratio of $0.1,0.2,0.3,0.4,0.6,1,2$ (The concentration of PhNO is $1 \times 10^{-4} \mathrm{~mol} / \mathrm{L}$ ).

The UV-vis spectroscopy was used to test the interaction of $\mathbf{3 a}$ and $[\mathrm{Pd}(\text { allyl }) \mathrm{Cl}]_{2}$. The typical absorption at $285 \mathrm{~nm}$ and $310 \mathrm{~nm}$ of nitrosobenzene both rose with bathochromic shift of $5 \mathrm{~nm}$ by adding $[\mathrm{Pd}(\text { allyl }) \mathrm{Cl}]_{2}$, and the typical peak at $260 \mathrm{~nm}$ of $[\mathrm{Pd}(\text { allyl }) \mathrm{Cl}]_{2}$ showed a hypsochromic shift about $5 \mathrm{~nm}$ with addition of 3 , which demonstrates that nitrosobenzene $\mathbf{3 a}$ could be activated by $[\mathrm{Pd}(\text { allyl }) \mathrm{Cl}]_{2}$ and the umpolung chemistry of the oxygen atom facilitated the designed reaction. 
5. Operando IR spectroscopy specie lines of the $[\mathrm{Pd}(\mathrm{allyl}) \mathrm{Cl}]_{2}$ and nitrosobenzene in the reaction mixture.

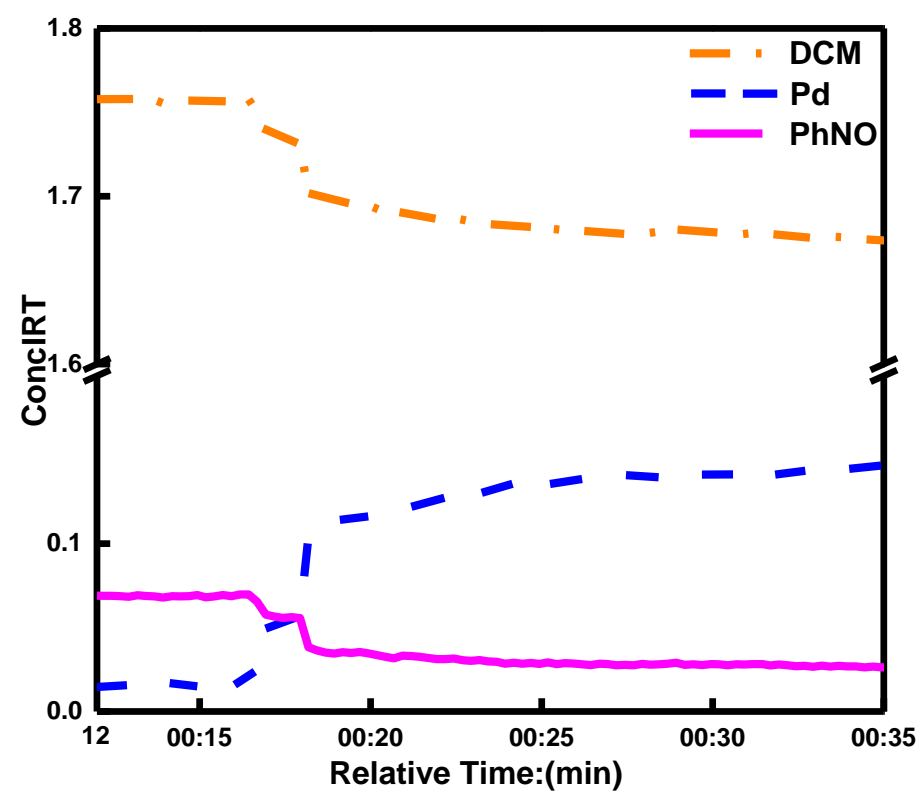

FigureS3. Operando IR spectroscopy specie lines of the $[\mathrm{Pd}(\text { allyl }) \mathrm{Cl}]_{2}$ and nitrosobenzene in the reaction mixture. The reaction was performed in a $0.1 \mathrm{mmol}$ scale of $\mathbf{3}$ and $[\mathrm{Pd}(\mathrm{allyl}) \mathrm{Cl}]_{2}$, respectively. 
6. ${ }^{1} \mathrm{HNMR}$ of the mixture of the $\left[\mathrm{Pd}(\text { allyl) } \mathrm{Cl}]_{2}\right.$ and nitrosobenzene in $\mathrm{CDCl}_{3}$ and DMSO

(a)

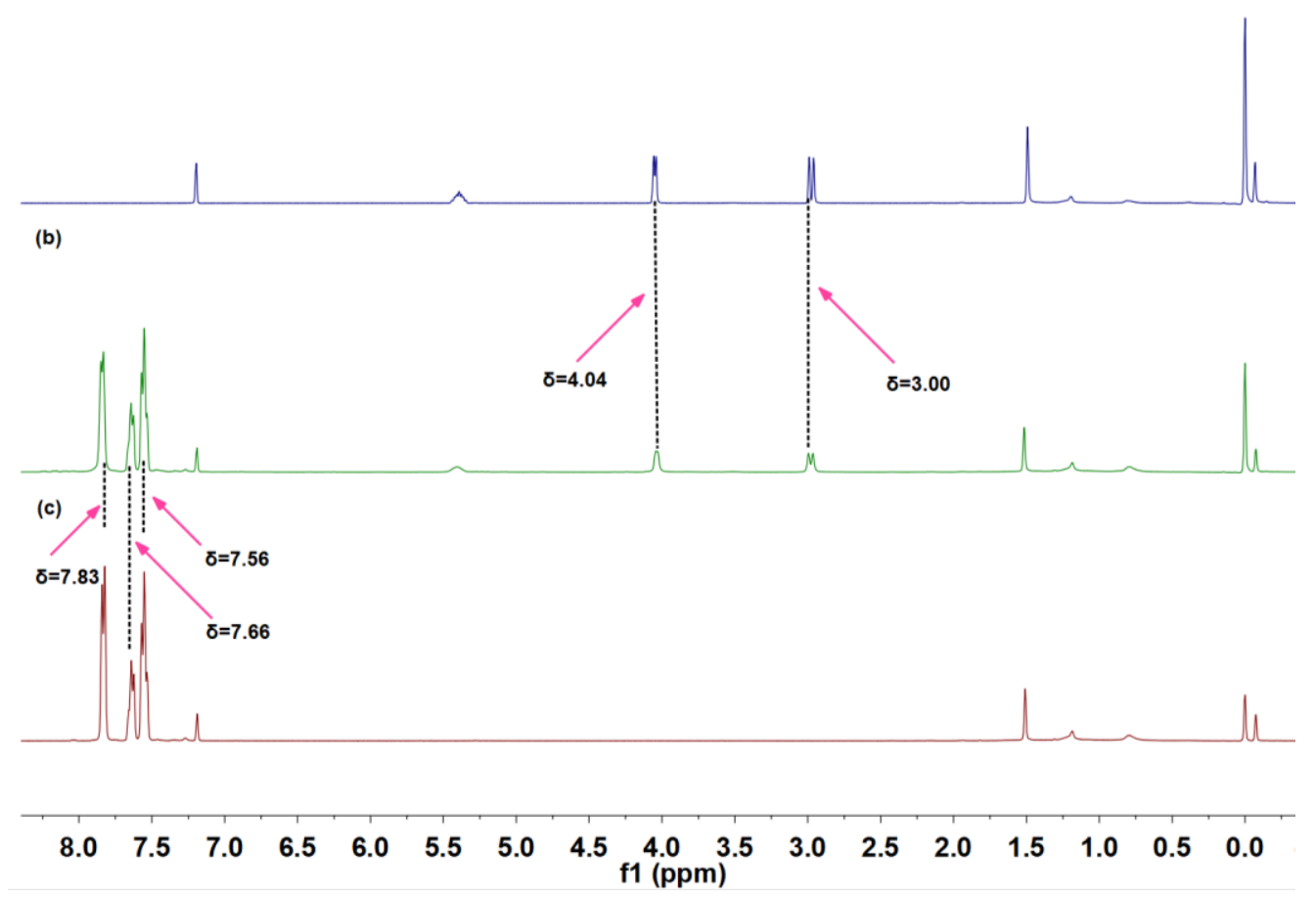

FigureS4 ${ }^{1} \mathrm{H}$ NMR Spectra of (a) $[\mathrm{Pd}(\text { allyl }) \mathrm{Cl}]_{2}$ 、 (b) the $0.1: 1$ mixture of the

$\left[\mathrm{Pd}(\text { allyl) } \mathrm{Cl}]_{2}\right.$ and $\mathrm{PhNO}$ (the concentration of $\left[\mathrm{Pd}(\text { allyl)Cl}]_{2}\right.$ is $0.02 \mathrm{~mol} / \mathrm{L}$ ) (c) $\mathrm{PhNO}$ in $\mathrm{CDCl}_{3}$ 


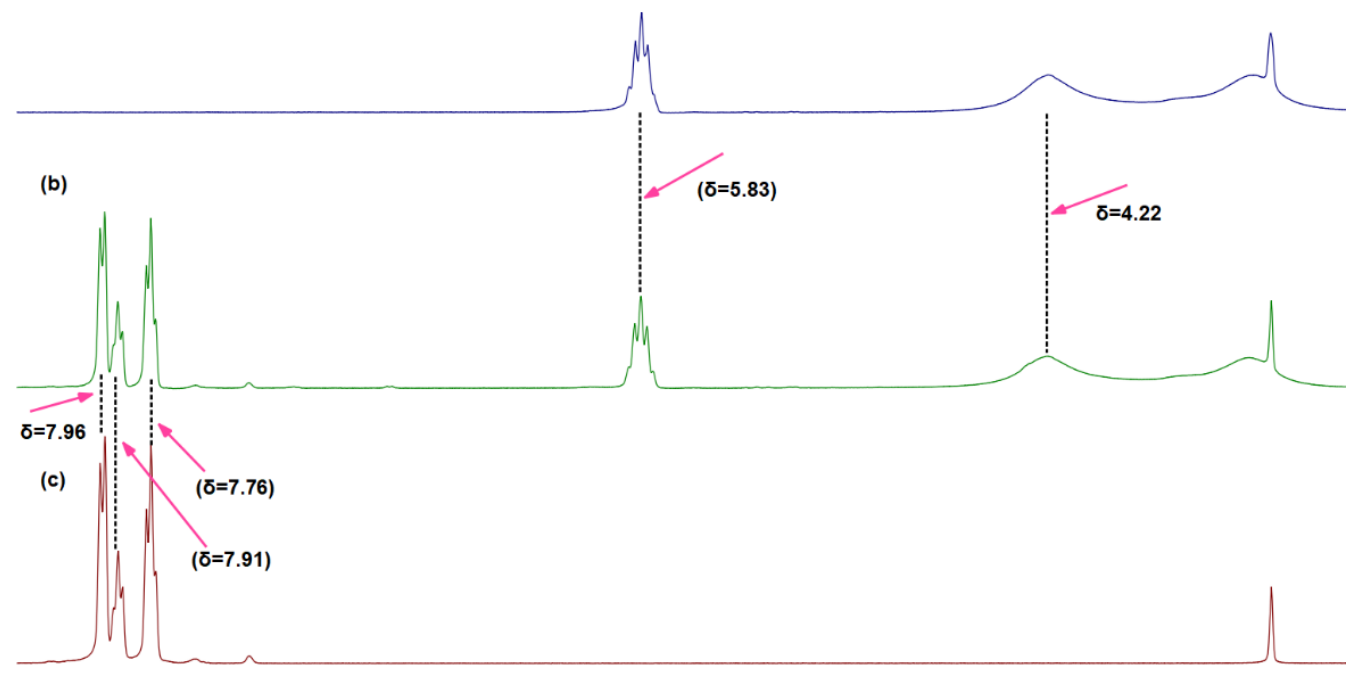

8.2 8.0 7.8 7.6 7.4 7.2 7.0 6.8 6.6 6.4 6.2 6.0 5.8 5.6 5.4 5.2 5.0 4.8 4.6 4.4 4.2 4.0 3.8 3.6 3.43 .2 f1 (ppm)

FigureS5 ${ }^{1} \mathrm{H}$ NMR Spectra of (a) $[\mathrm{Pd}(\text { allyl }) \mathrm{Cl}]_{2}$ 、(b) the $1: 1$ mixture of the $[\mathrm{Pd}(\text { allyl }) \mathrm{Cl}]_{2}$ and $\mathrm{PhNO}$ (the concentration of $[\mathrm{Pd}(\text { allyl }) \mathrm{Cl}]_{2}$ is $\left.0.1 \mathrm{~mol} / \mathrm{L}\right)(\mathrm{c}) \mathrm{PhNO}$ in DMSO 


\section{X-ray Diffraction Parameters and Data of $4 p$}

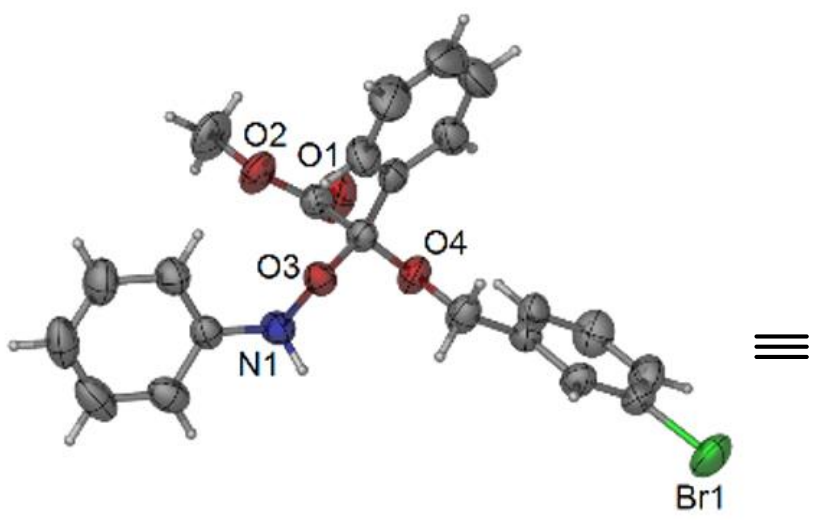

Bond precision:

Cell:

Temperature:

Space group

Hall group

Moiety formula

Sum formula

$\mathrm{Mr}$

Dx, g cm-3

Z

$\mathrm{Mu}(\mathrm{mm}-1)$

F000

F000'

h,k,lmax

Nref

Tmin,Tmax

Tmin'
$\mathrm{C}-\mathrm{C}=0.0056 \mathrm{~A}$ $\mathrm{a}=15.8641(11) \mathrm{b}=11.8005(8) \quad \mathrm{c}=11.7015(8)$ alpha $=90 \quad$ beta $=107.853(2)$ gamma $=90$ $296 \mathrm{~K}$

Calculated 2085.1(2)

$\mathrm{P} 21 / \mathrm{c}$

$-\mathrm{P} 2 \mathrm{ybc}$

$\mathrm{C} 22 \mathrm{H} 20 \mathrm{Br} \mathrm{N} \mathrm{O} 4$

$\mathrm{C} 22 \mathrm{H} 20 \mathrm{Br} \mathrm{N} \mathrm{O} 4$

442.29

1.409

4

1.998

904.0

903.25

$18,14,13$

3671

$0.418,0.619$

0.369

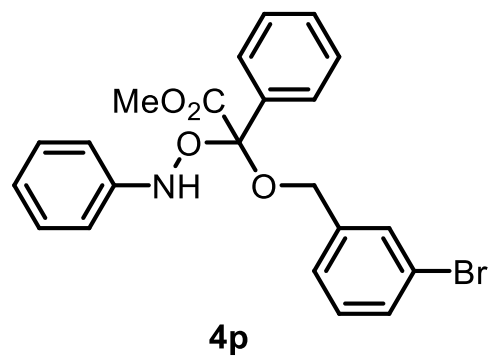

Wavelength $=0.71073$

Correction method $=$ \# Reported T Limits: Tmin $=0.447$ Tmax $=0.646$ AbsCorr $=$ MULTI-SCAN

Data completeness $=0.999$ $\operatorname{Theta}(\max )=25.010$

$\mathrm{R}($ reflections $)=0.0429(2492)$ $w R 2($ reflections $)=0.1118(3668)$

$S=0.982$ Npar $=257$

Figure S6 The single crystal analysis for $4 p$ (CCDC number: 1984265)

The ellipsoid contour of probability levels is $50 \%$. 
Scheme S2 ORTEP plot of 4p

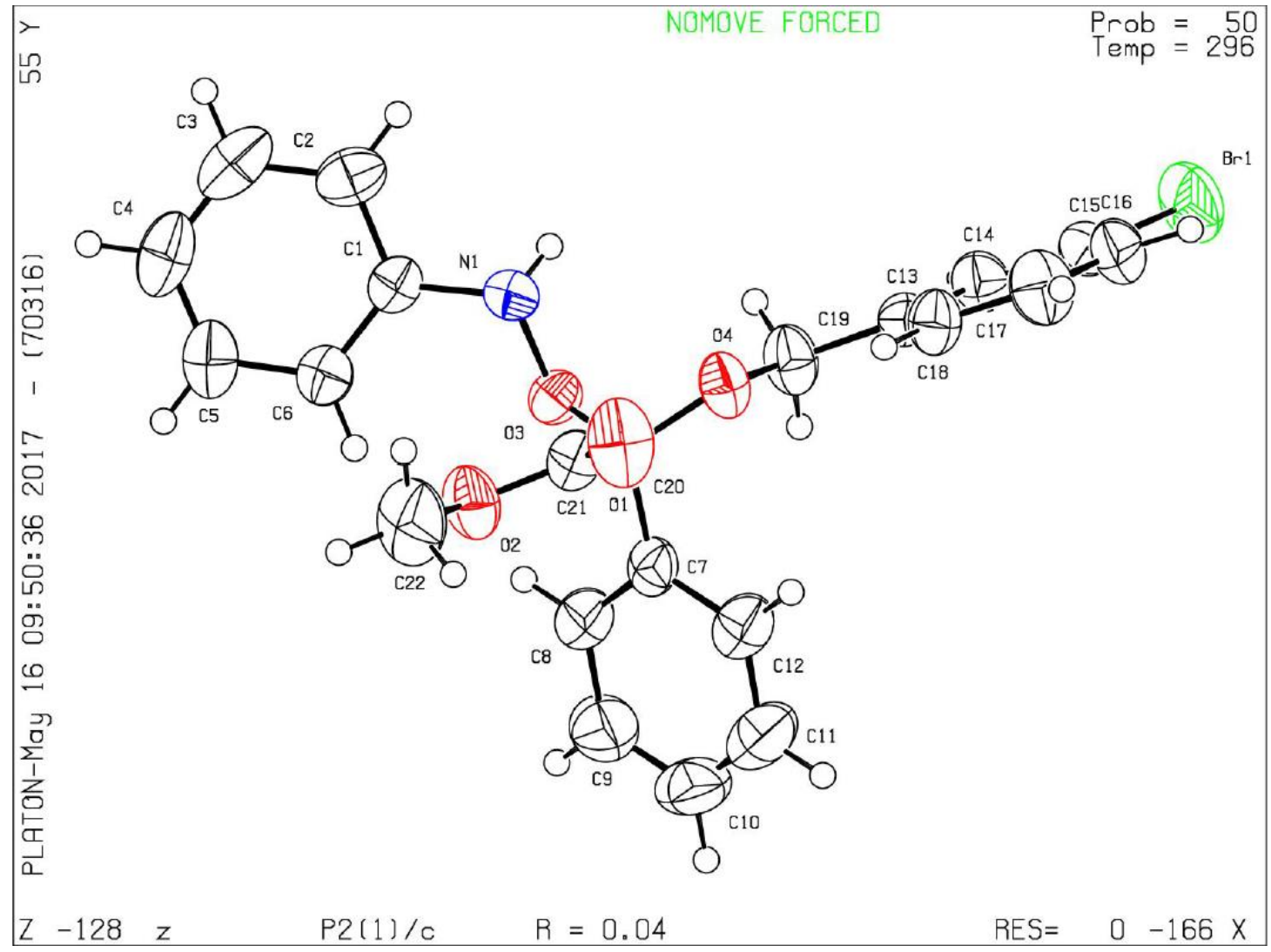

\section{Preparation Procedure for Single Crystal Sample of $4 p$}

In a $20 \mathrm{~mL}$ test glass bottle, a solution of compound $\mathbf{4 d}(20 \mathrm{mg})$ in EA $(1 \mathrm{~mL})$ was added in one portion after filtration. Then PE $(20 \mathrm{~mL})$ was added slowly along the wall of the test bottle in order to keep the PE and EA solution separated into two layer. Then left the test bottle standing at the room temperature until the single crystal was formed. 


\section{NMR Spectra of Compounds}

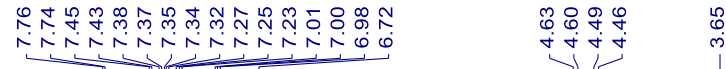

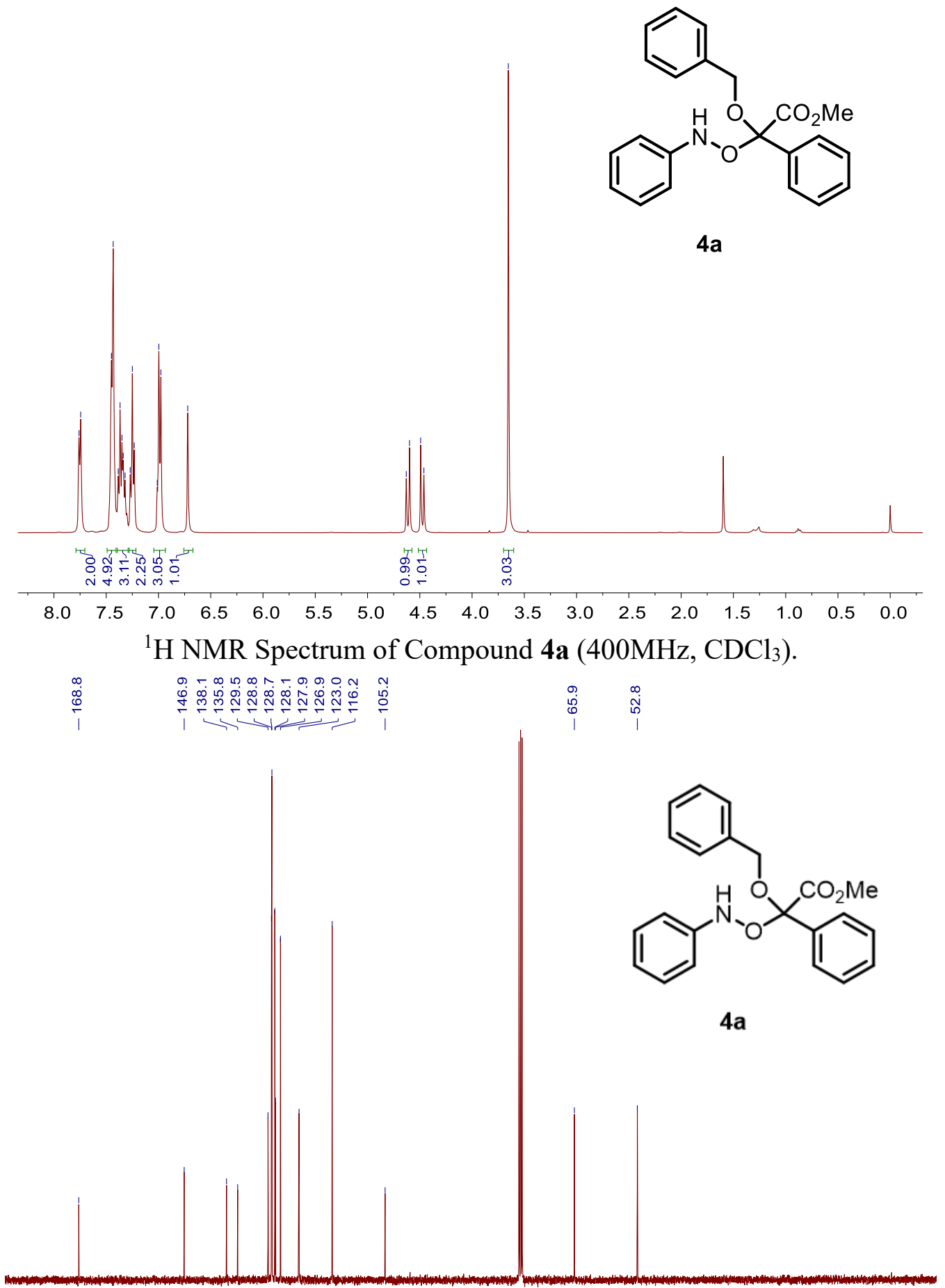

$\begin{array}{llllllllllllllllllll}180 & 170 & 160 & 150 & 140 & 130 & 120 & 110 & 100 & 90 & 80 & 70 & 60 & 50 & 40 & 30 & 20 & 10 & 0 & -\end{array}$

${ }^{13} \mathrm{C}\left\{{ }^{1} \mathrm{H}\right\} \mathrm{NMR}$ Spectrum of Compound $4 \mathrm{a}\left(100 \mathrm{MHz}, \mathrm{CDCl}_{3}\right)$. 


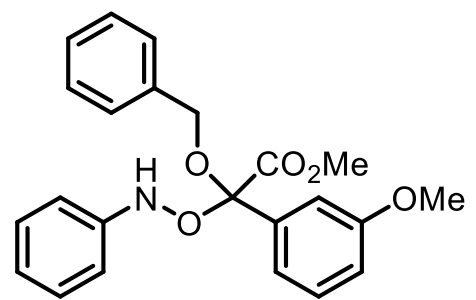

4b
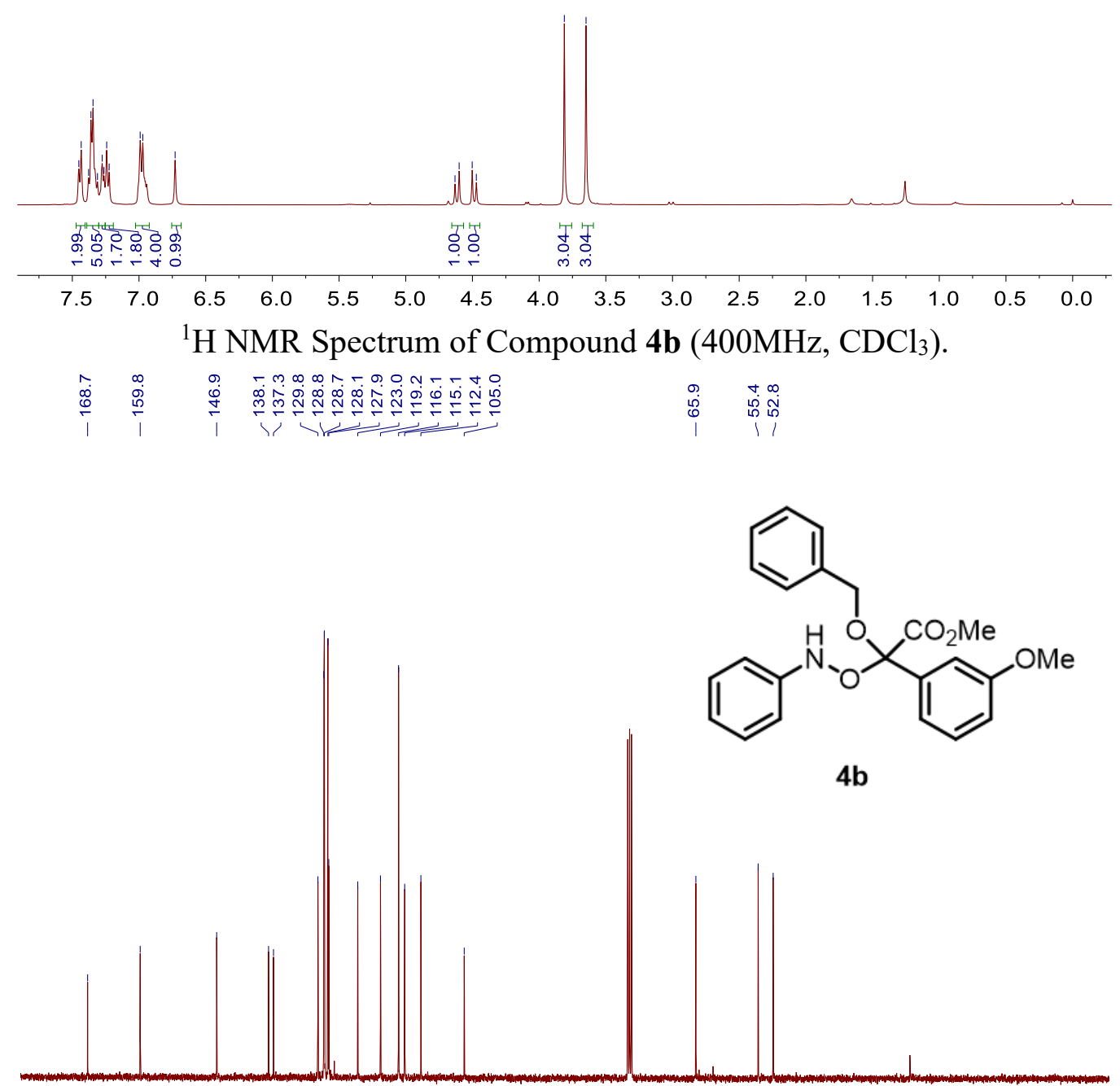

$\begin{array}{lllllllllllllllllll}80 & 170 & 160 & 150 & 140 & 130 & 120 & 110 & 100 & 90 & 80 & 70 & 60 & 50 & 40 & 30 & 20 & 10 & 0\end{array}$

${ }^{13} \mathrm{C}\left\{{ }^{1} \mathrm{H}\right\} \mathrm{NMR}$ Spectrum of Compound $\mathbf{4 b}\left(100 \mathrm{MHz}, \mathrm{CDCl}_{3}\right)$. 

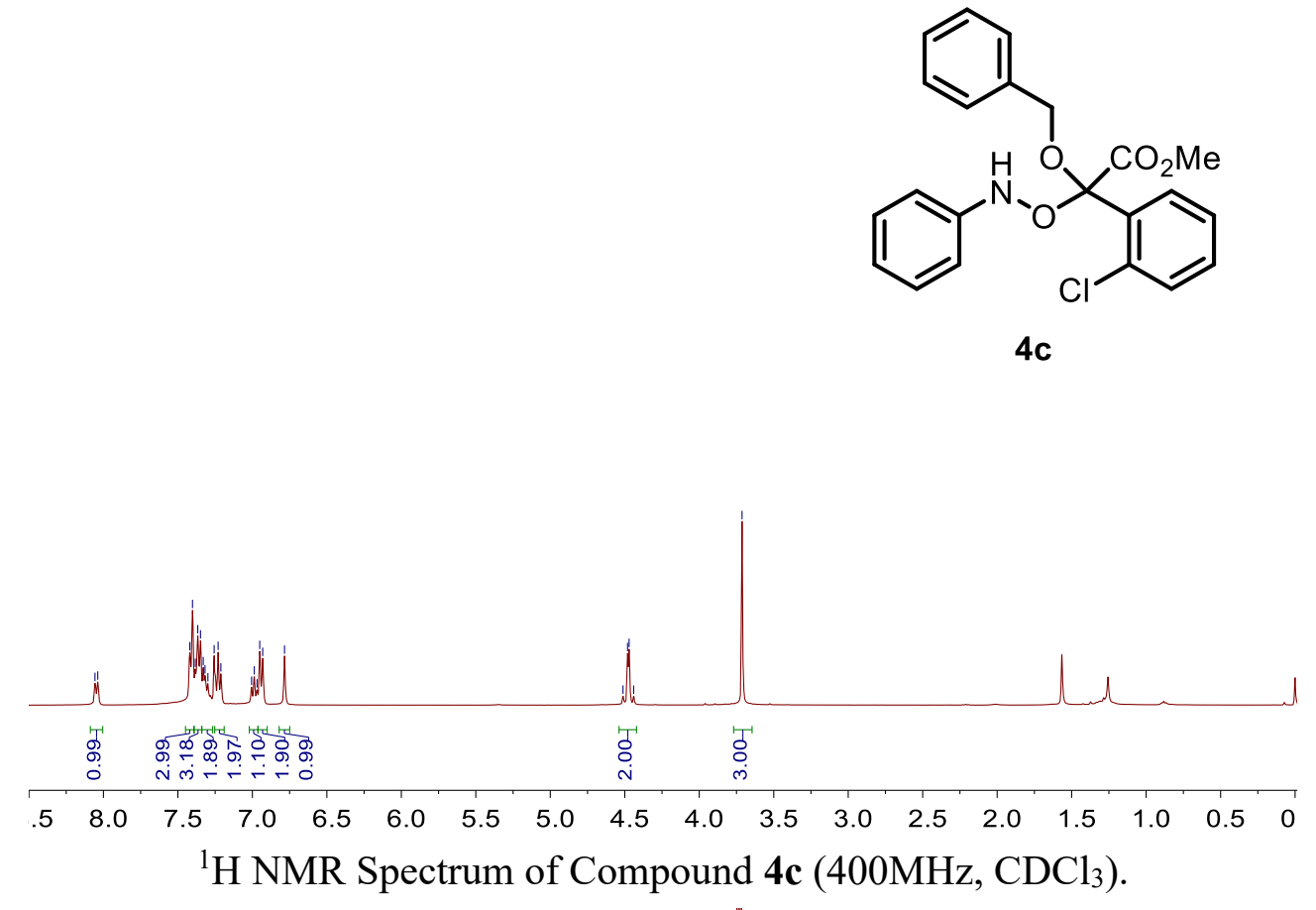

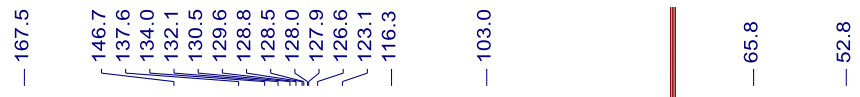<smiles>COC(OCc1ccccc1)(ONc1ccccc1)c1ccccc1Cl</smiles>

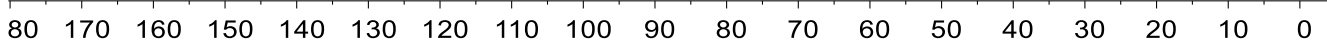

${ }^{13} \mathrm{C}\left\{{ }^{1} \mathrm{H}\right\} \mathrm{NMR}$ Spectrum of Compound $4 \mathbf{c}\left(100 \mathrm{MHz}, \mathrm{CDCl}_{3}\right)$. 

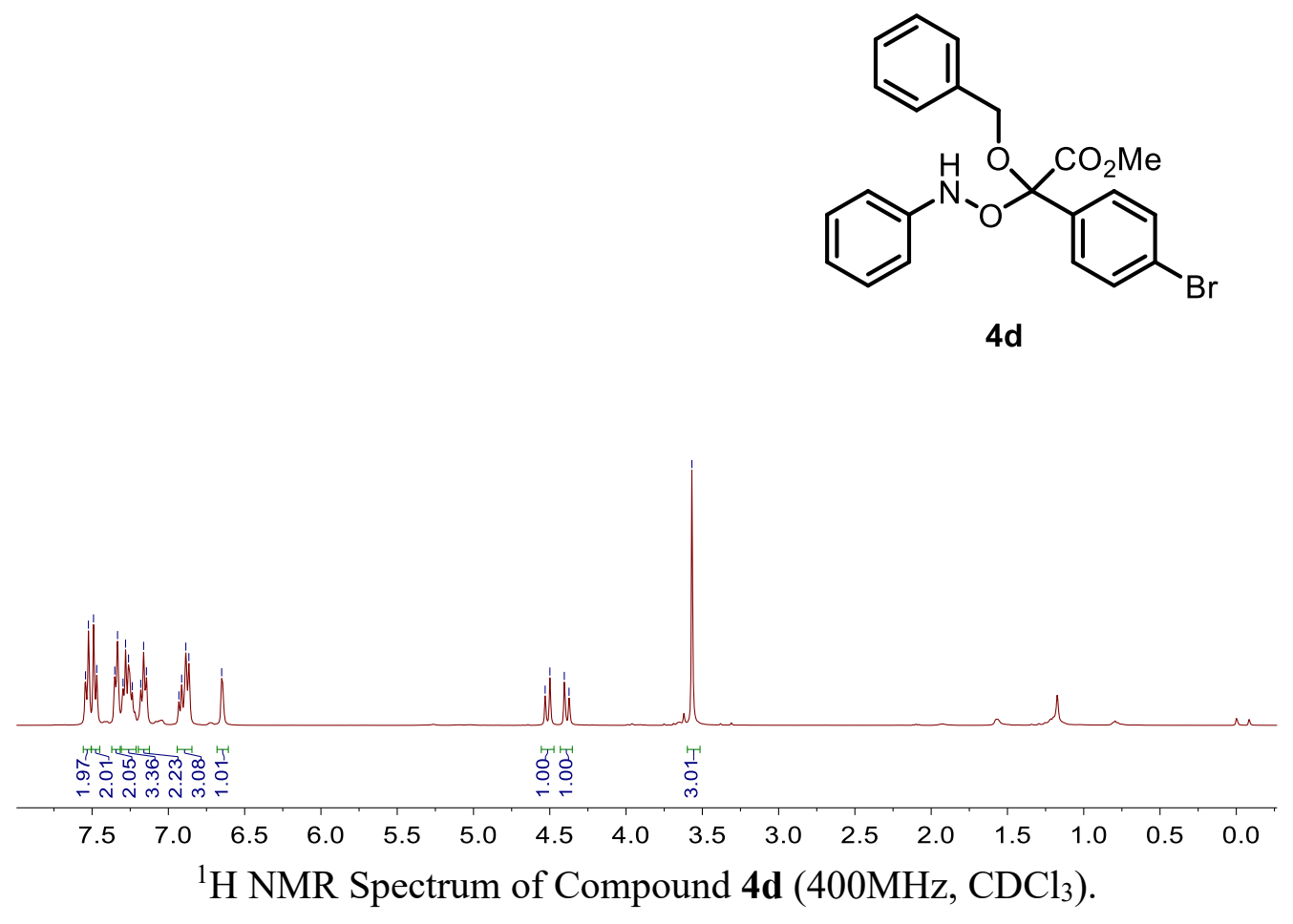

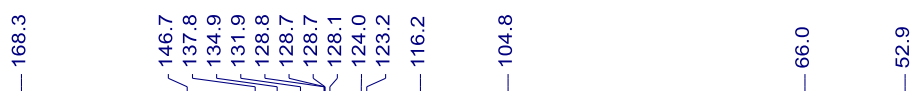

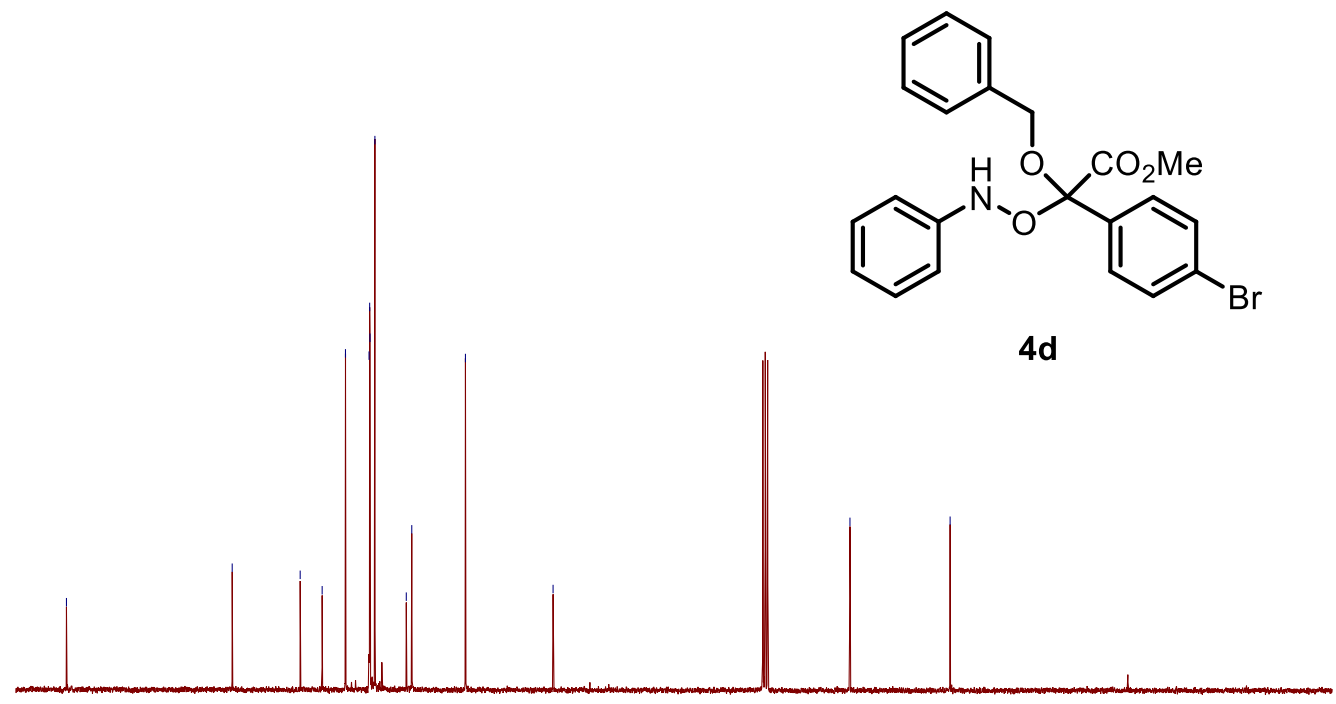

4d

$\begin{array}{lllllllllllllllll}170 & 160 & 150 & 140 & 130 & 120 & 110 & 100 & 90 & 80 & 70 & 60 & 50 & 40 & 30 & 20 & 10\end{array}$ ${ }^{13} \mathrm{C}\left\{{ }^{1} \mathrm{H}\right\} \mathrm{NMR}$ Spectrum of Compound 4d (100MHz, $\left.\mathrm{CDCl}_{3}\right)$. 


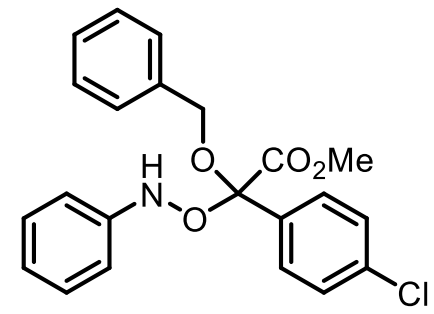

$4 \mathrm{e}$
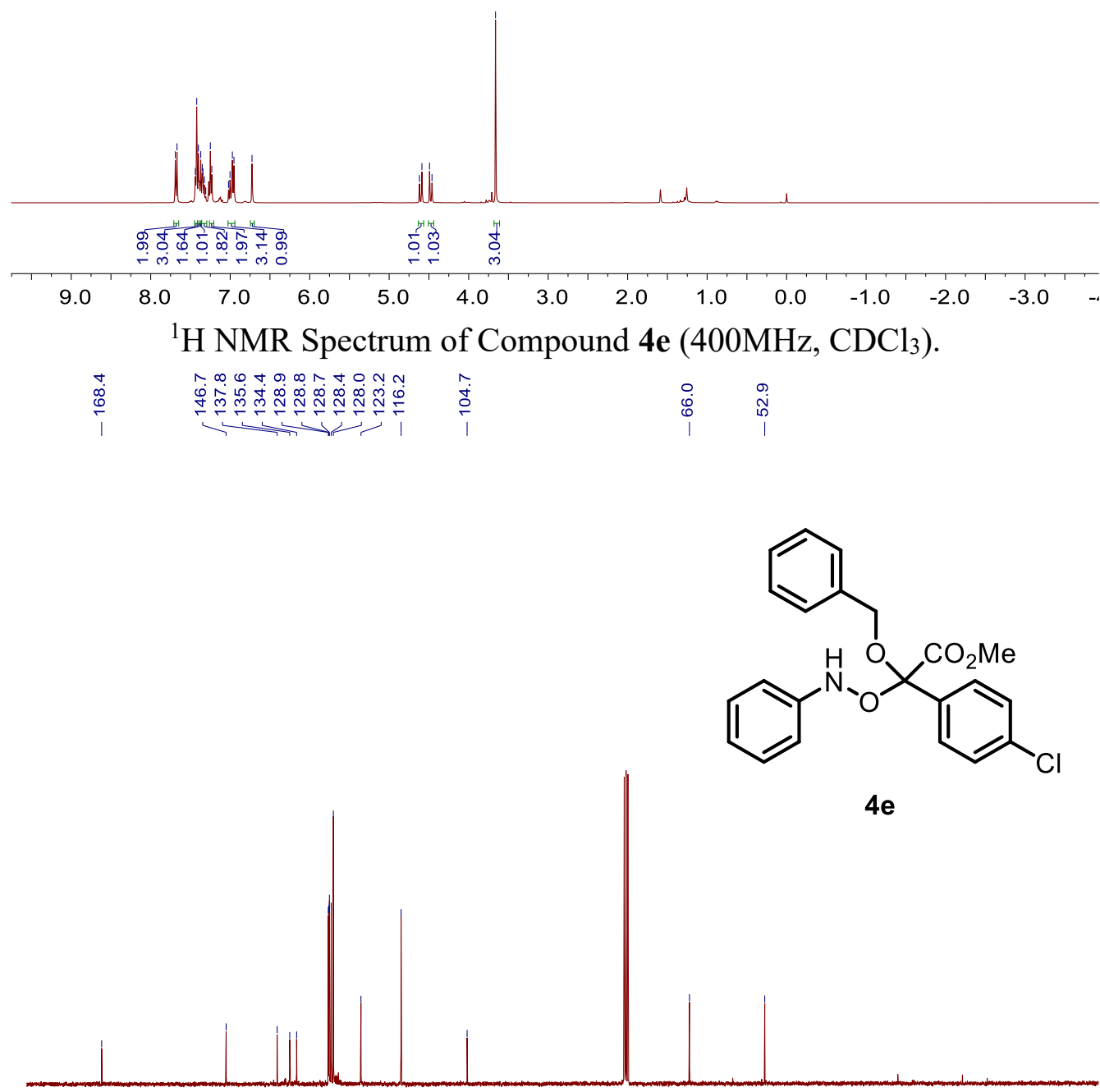

$\begin{array}{lllllllllllllllllll}80 & 170 & 160 & 150 & 140 & 130 & 120 & 110 & 100 & 90 & 80 & 70 & 60 & 50 & 40 & 30 & 20 & 10 & 0\end{array}$

${ }^{13} \mathrm{C}\left\{{ }^{1} \mathrm{H}\right\} \mathrm{NMR}$ Spectrum of Compound $4 \mathbf{e}\left(100 \mathrm{MHz}, \mathrm{CDCl}_{3}\right)$. 


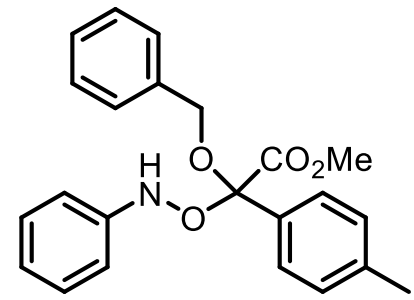

$4 \mathbf{f}$
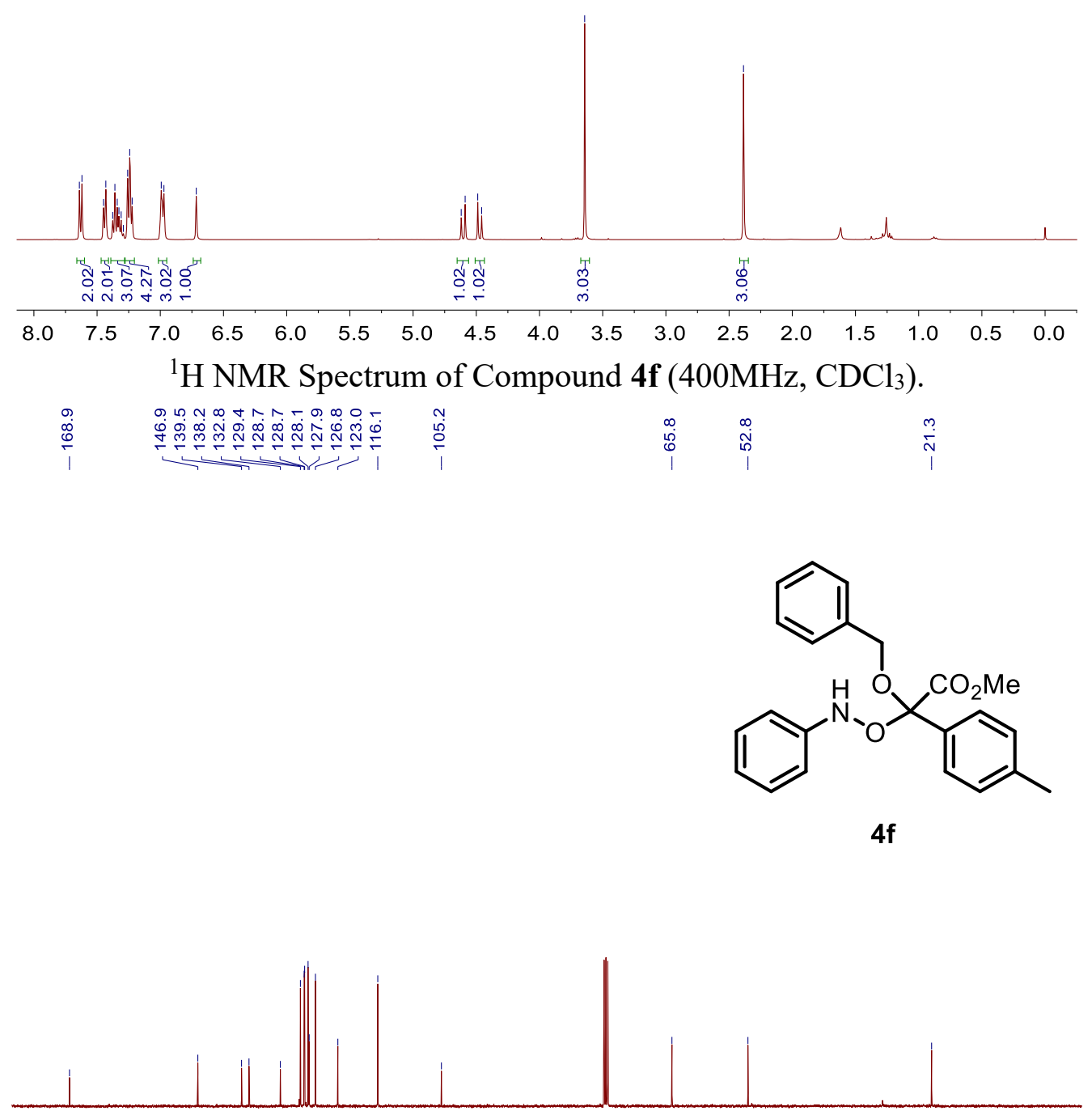

$\begin{array}{llllllllllllllllll}170 & 160 & 150 & 140 & 130 & 120 & 110 & 100 & 90 & 80 & 70 & 60 & 50 & 40 & 30 & 20 & 10 & 0\end{array}$

${ }^{13} \mathrm{C}\left\{{ }^{1} \mathrm{H}\right\} \mathrm{NMR}$ Spectrum of Compound $4 \mathbf{f}\left(100 \mathrm{MHz}, \mathrm{CDCl}_{3}\right)$. 


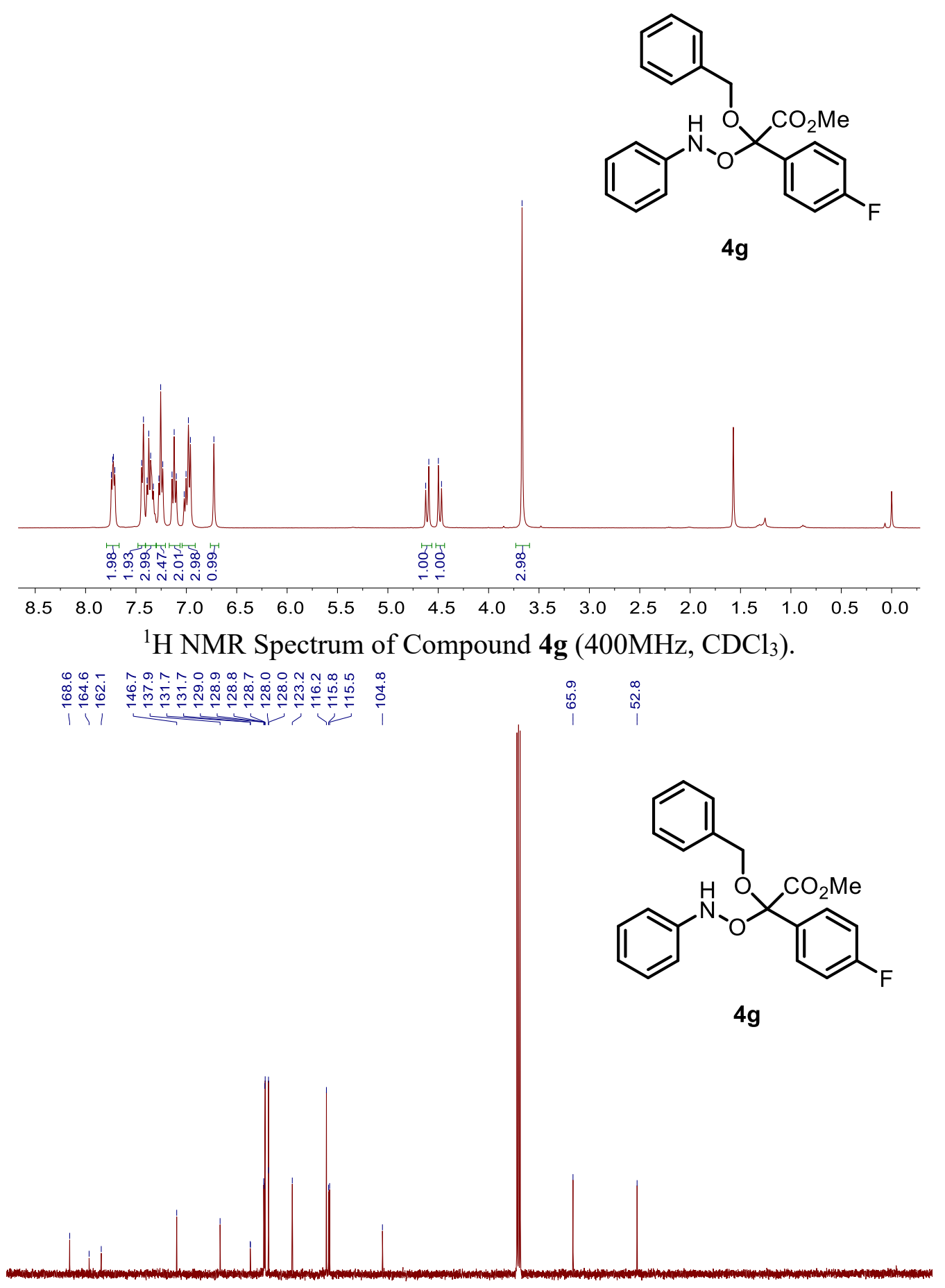

$\begin{array}{lllllllllllllllllll}180 & 170 & 160 & 150 & 140 & 130 & 120 & 110 & 100 & 90 & 80 & 70 & 60 & 50 & 40 & 30 & 20 & 10 & 0\end{array}$

${ }^{13} \mathrm{C}\left\{{ }^{1} \mathrm{H}\right\}$ NMR Spectrum of Compound $\mathbf{4 g}\left(100 \mathrm{MHz}, \mathrm{CDCl}_{3}\right)$. 


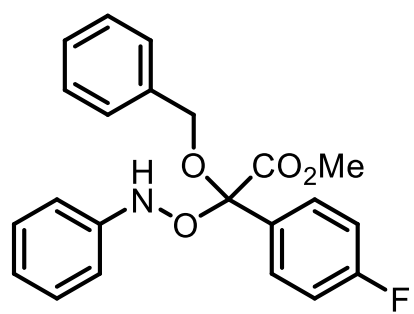

$4 g$

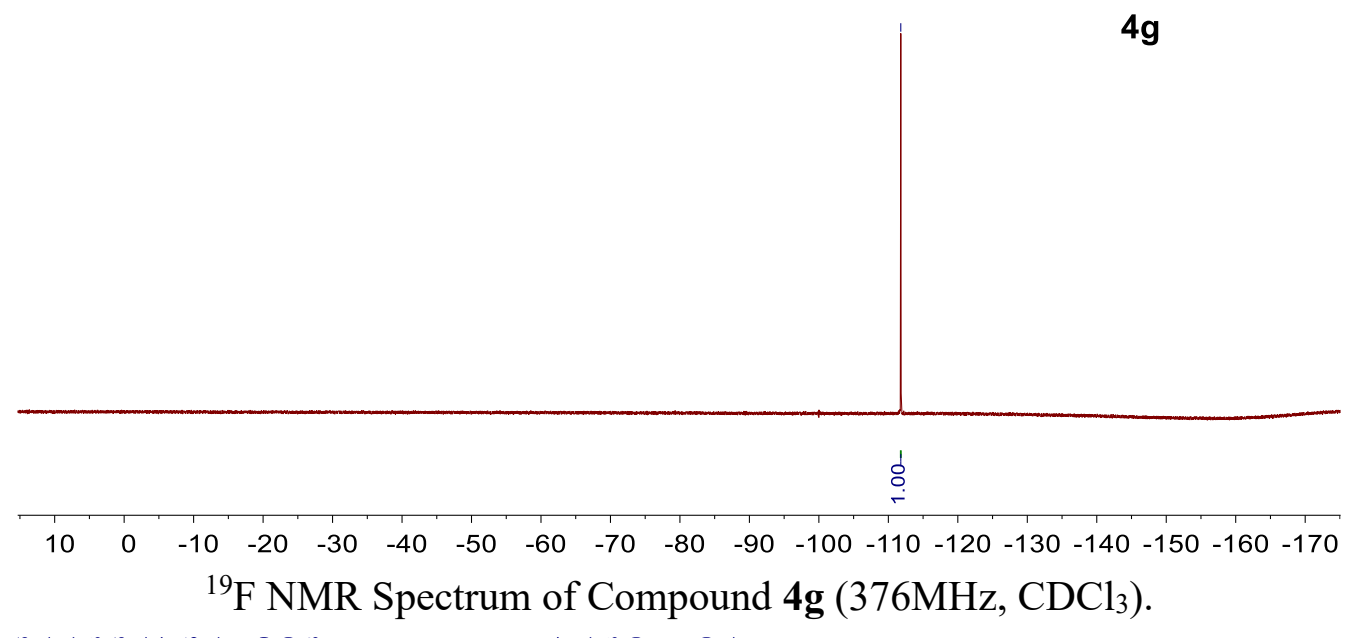

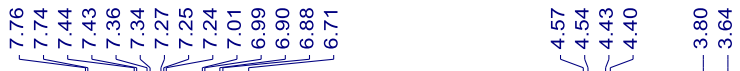

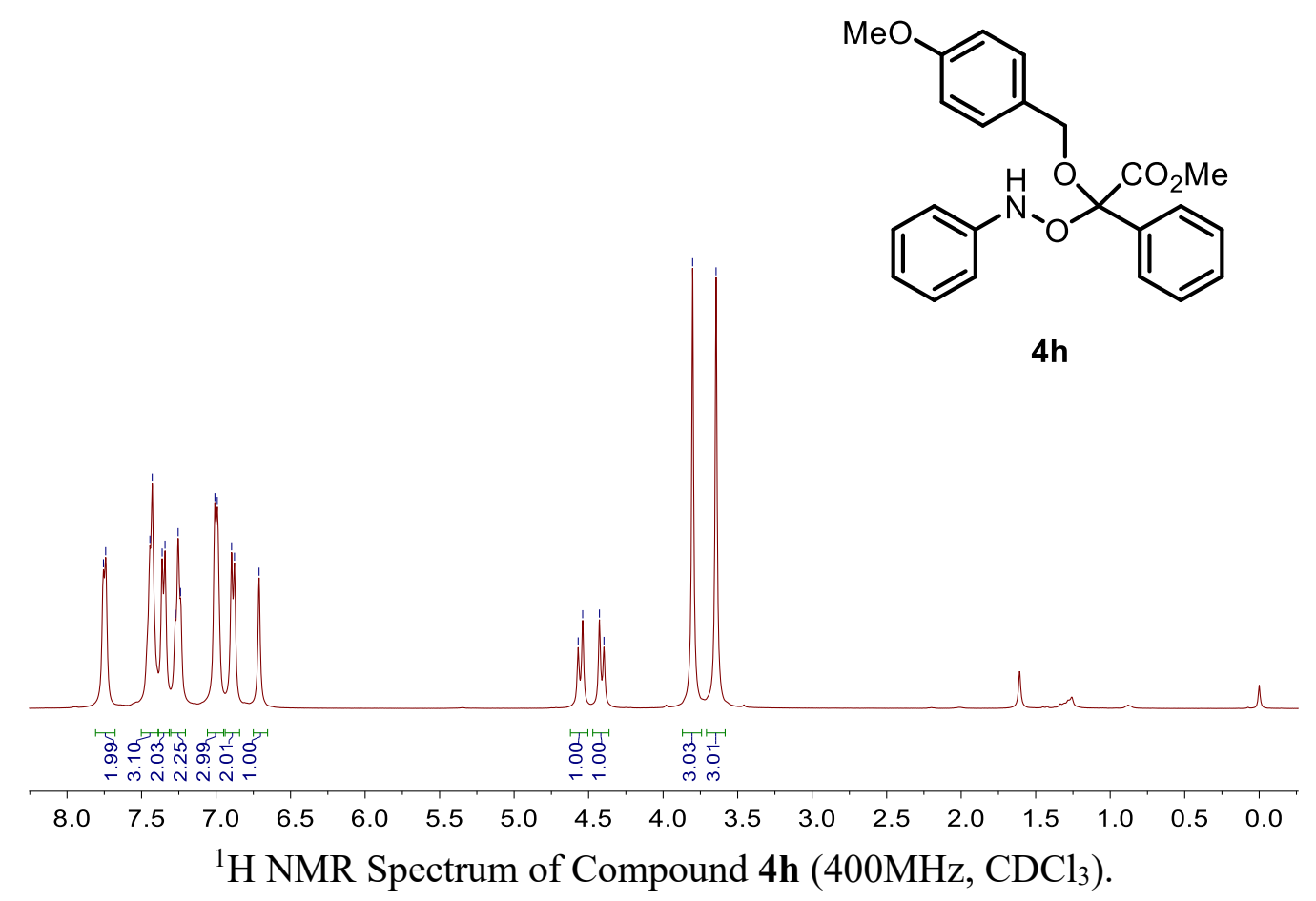


$\mathrm{MeO}$<smiles>COC(=O)C(OCc1ccc(OC)cc1)(ONc1ccccc1)c1ccccc1</smiles>

4h

$21020019018017016015014013012011010090 \quad 80 \quad 70 \quad 60 \quad 50 \quad 40 \quad 30 \quad 20 \quad 10 \quad 0 \quad-10$

${ }^{13} \mathrm{C}\left\{{ }^{1} \mathrm{H}\right\} \mathrm{NMR}$ Spectrum of Compound $\mathbf{4 h}\left(100 \mathrm{MHz}, \mathrm{CDCl}_{3}\right)$.

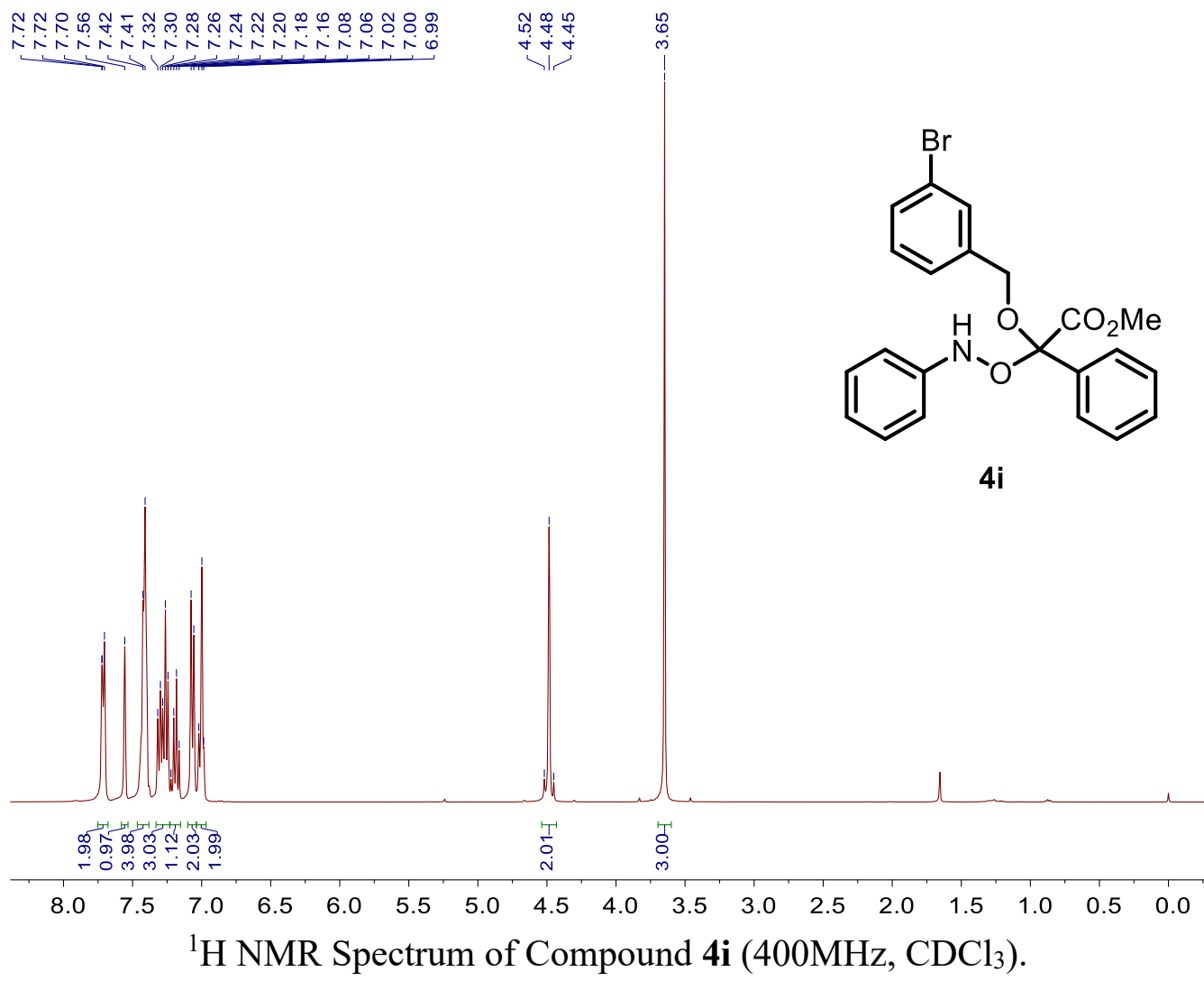




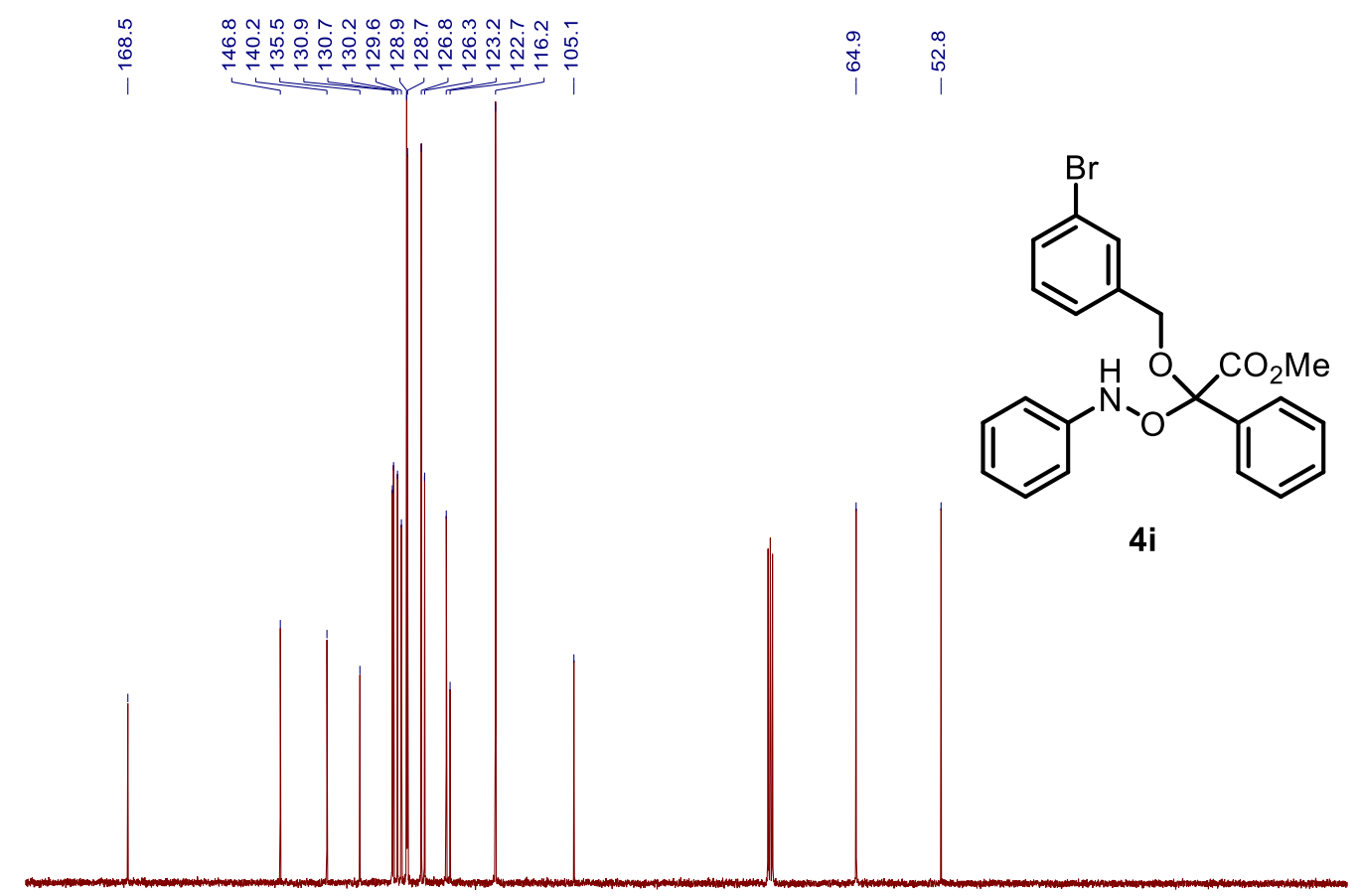

$\begin{array}{lllllllllllllllllll}180 & 170 & 160 & 150 & 140 & 130 & 120 & 110 & 100 & 90 & 80 & 70 & 60 & 50 & 40 & 30 & 20 & 10 & 0\end{array}$

${ }^{13} \mathrm{C}\left\{{ }^{1} \mathrm{H}\right\} \mathrm{NMR}$ Spectrum of Compound $4 \mathbf{i}\left(100 \mathrm{MHz}, \mathrm{CDCl}_{3}\right)$.

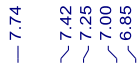

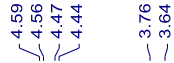

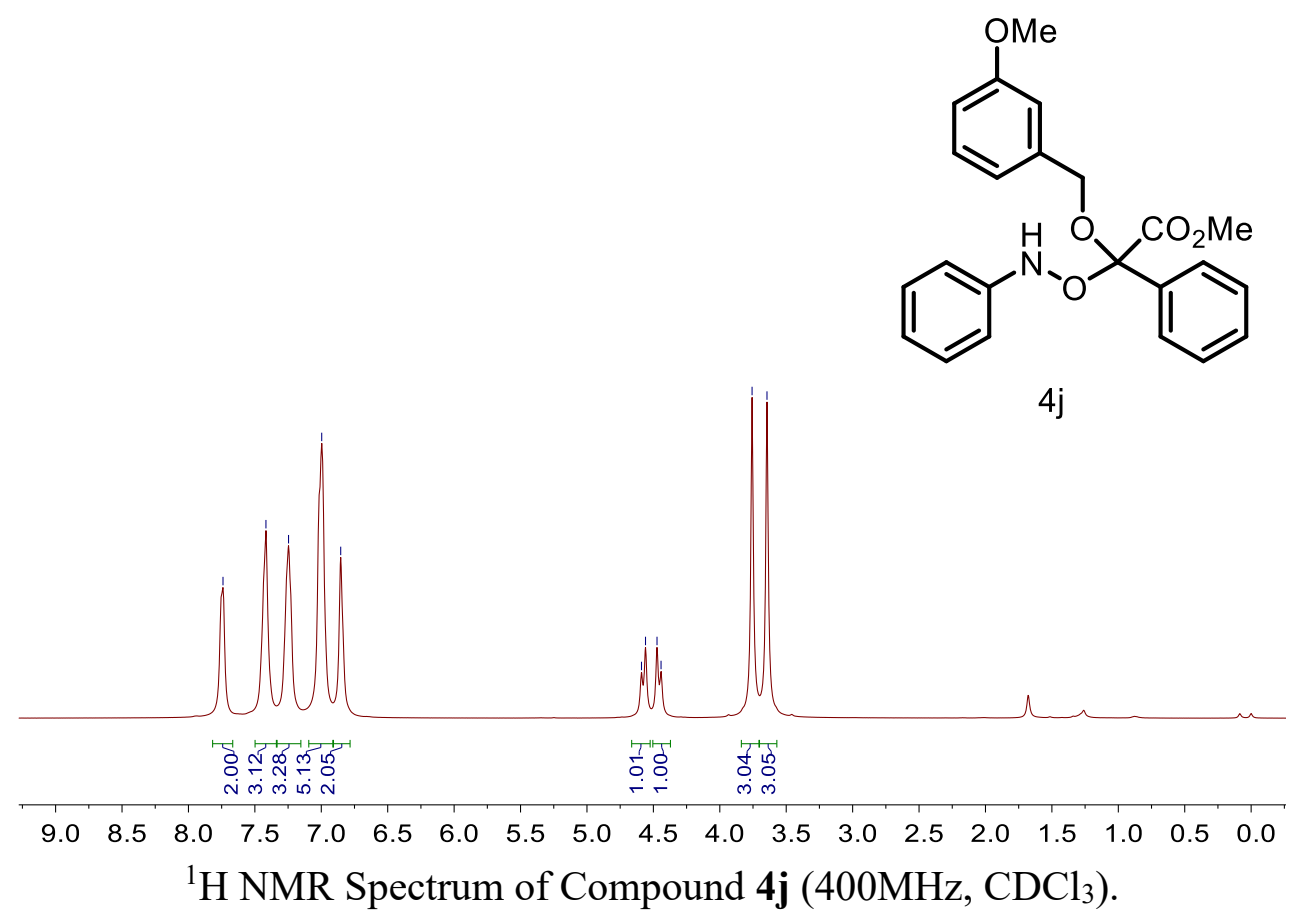




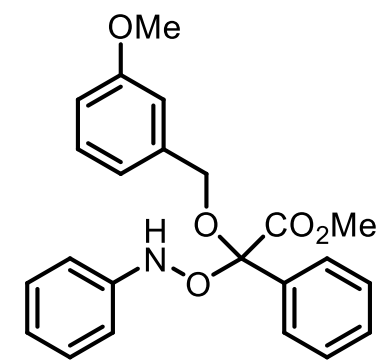

$4 j$

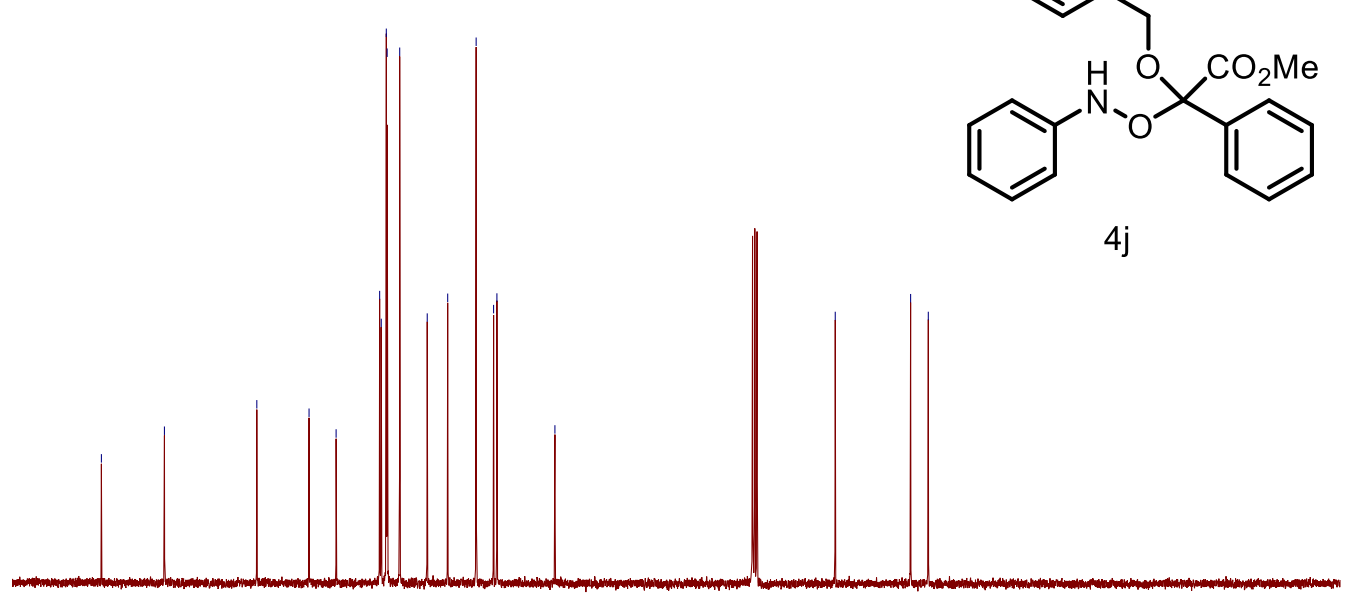

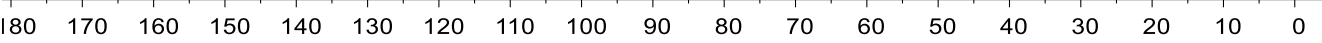

${ }^{13} \mathrm{C}\left\{{ }^{1} \mathrm{H}\right\} \mathrm{NMR}$ Spectrum of Compound $\mathbf{4 j}\left(100 \mathrm{MHz}, \mathrm{CDCl}_{3}\right)$.

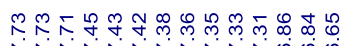

rinkrisingo

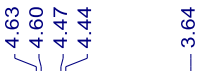

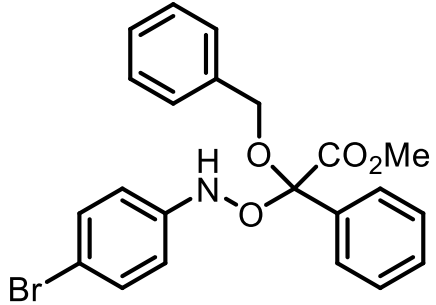

4k

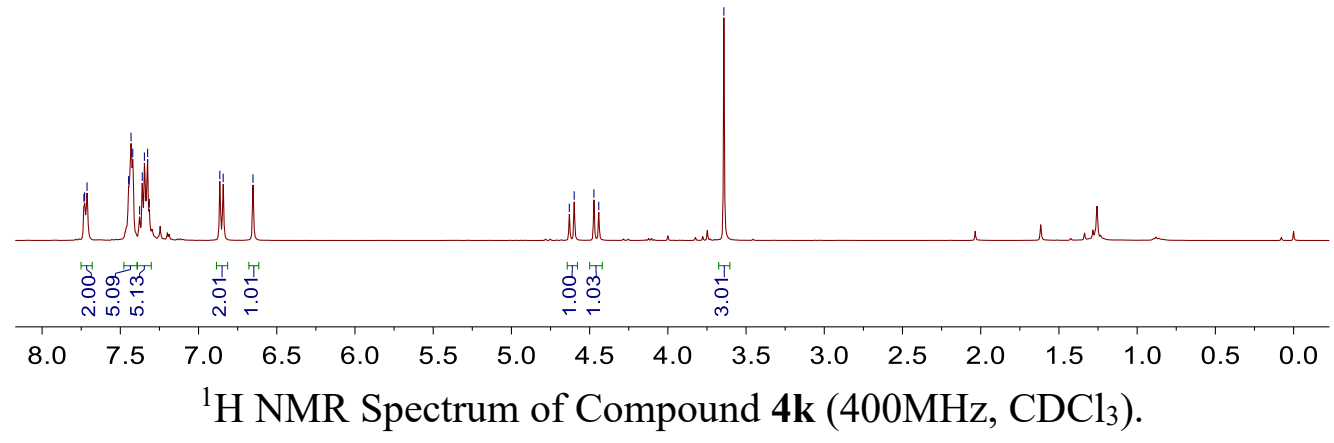




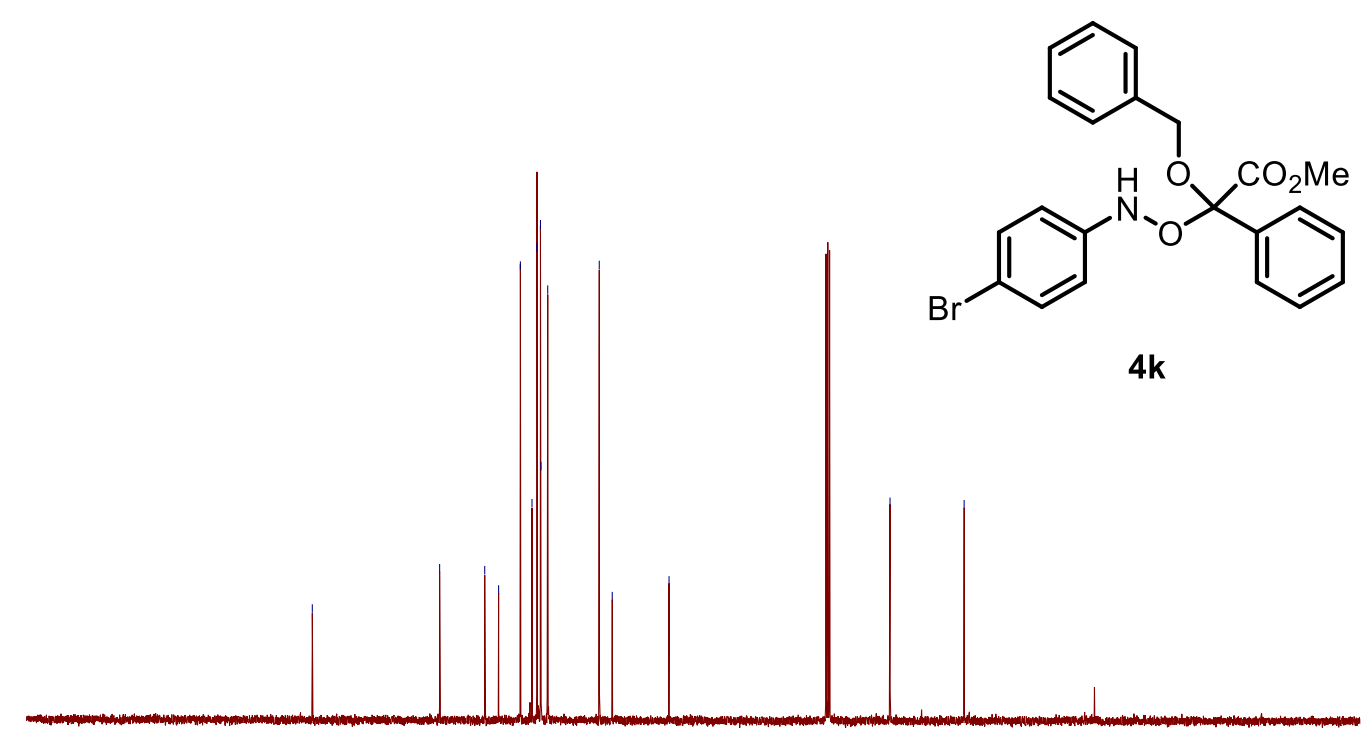

$21020019018017016015014013012011010090 \quad 80 \quad 70 \quad 60 \quad 50 \quad 40 \quad 30 \quad 20 \quad 10 \quad 0 \quad-10$

${ }^{13} \mathrm{C}\left\{{ }^{1} \mathrm{H}\right\} \mathrm{NMR}$ Spectrum of Compound $4 \mathbf{k}\left(100 \mathrm{MHz}, \mathrm{CDCl}_{3}\right)$.

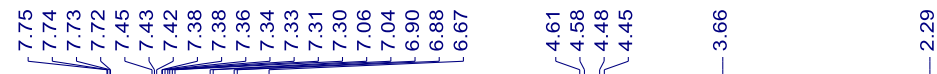

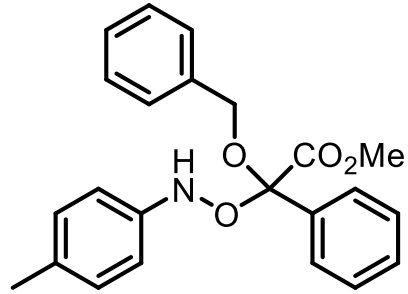

4I

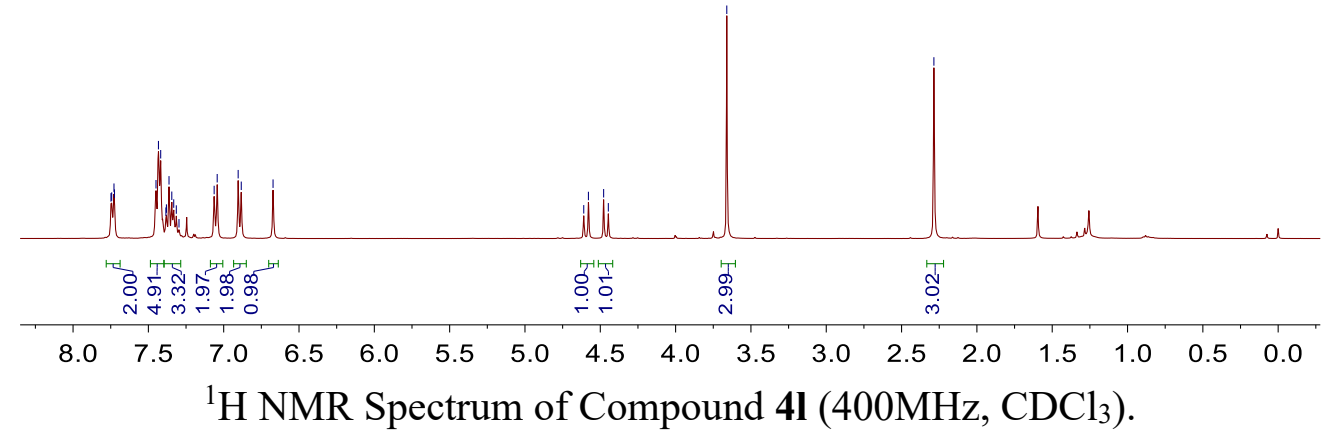




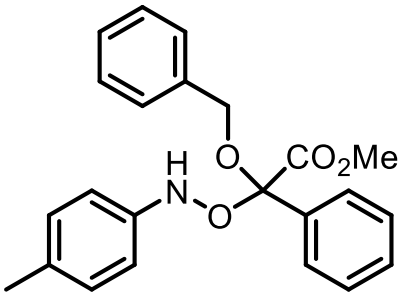

4I

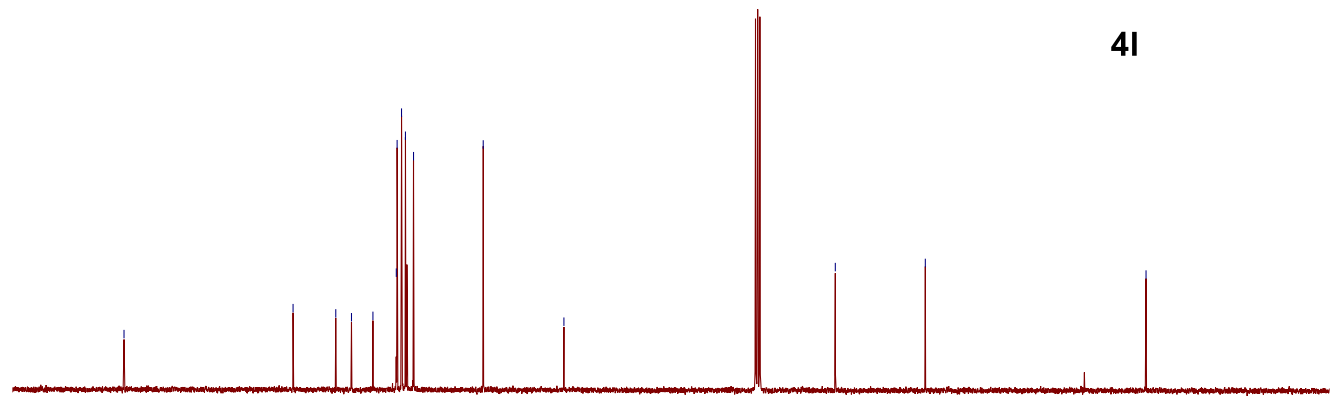

$\begin{array}{lllllllllllllllllll}180 & 170 & 160 & 150 & 140 & 130 & 120 & 110 & 100 & 90 & 80 & 70 & 60 & 50 & 40 & 30 & 20 & 10 & 0\end{array}$

${ }^{13} \mathrm{C}\left\{{ }^{1} \mathrm{H}\right\} \mathrm{NMR}$ Spectrum of Compound $4 \mathbf{l}\left(100 \mathrm{MHz}, \mathrm{CDCl}_{3}\right)$.

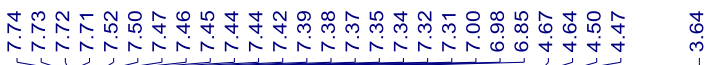<smiles>COC(OCc1ccccc1)(ONc1ccc(C#N)cc1)c1ccccc1</smiles>

$4 m$

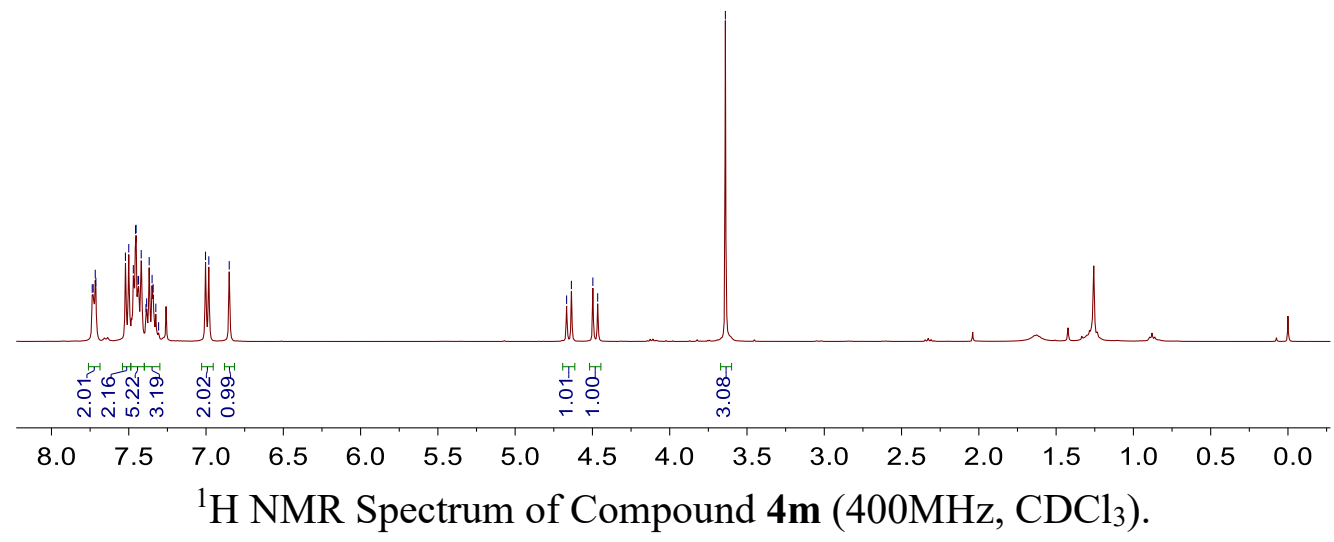




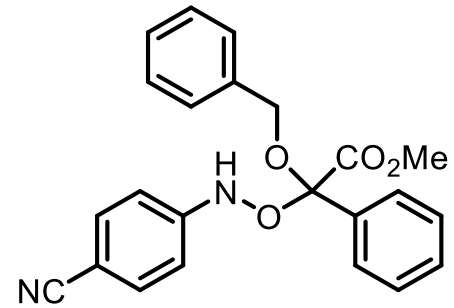

$4 \mathrm{~m}$

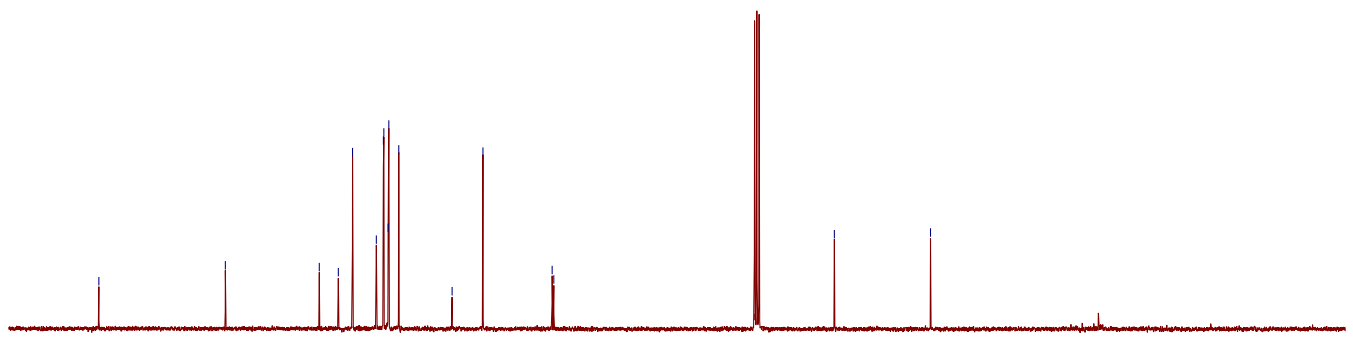

$\begin{array}{lllllllllllllllllll}80 & 170 & 160 & 150 & 140 & 130 & 120 & 110 & 100 & 90 & 80 & 70 & 60 & 50 & 40 & 30 & 20 & 10 & 0\end{array}$

${ }^{13} \mathrm{C}\left\{{ }^{1} \mathrm{H}\right\} \mathrm{NMR}$ Spectrum of Compound $4 \mathbf{m}\left(100 \mathrm{MHz}, \mathrm{CDCl}_{3}\right)$.

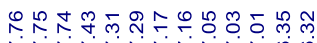

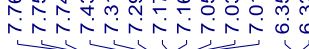

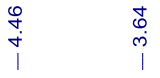

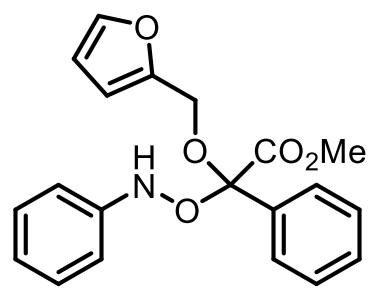

$4 n$

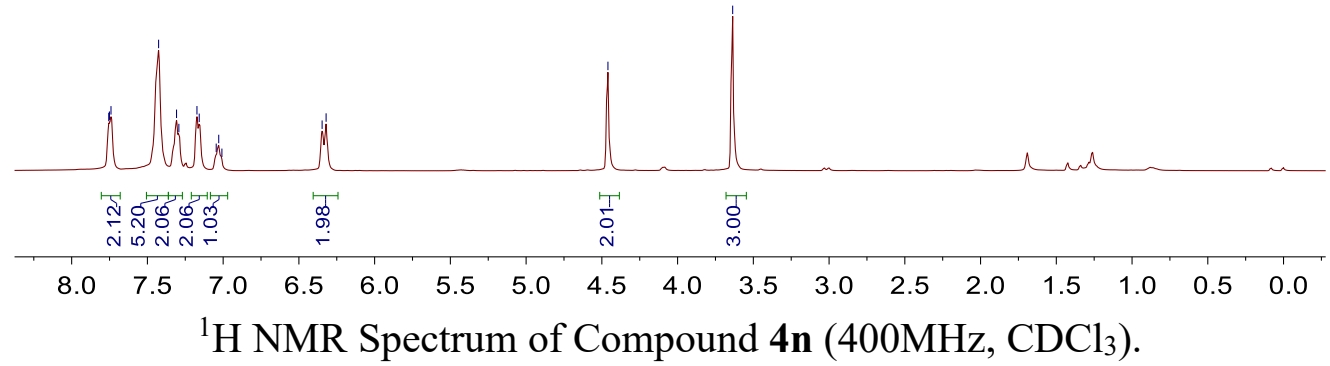




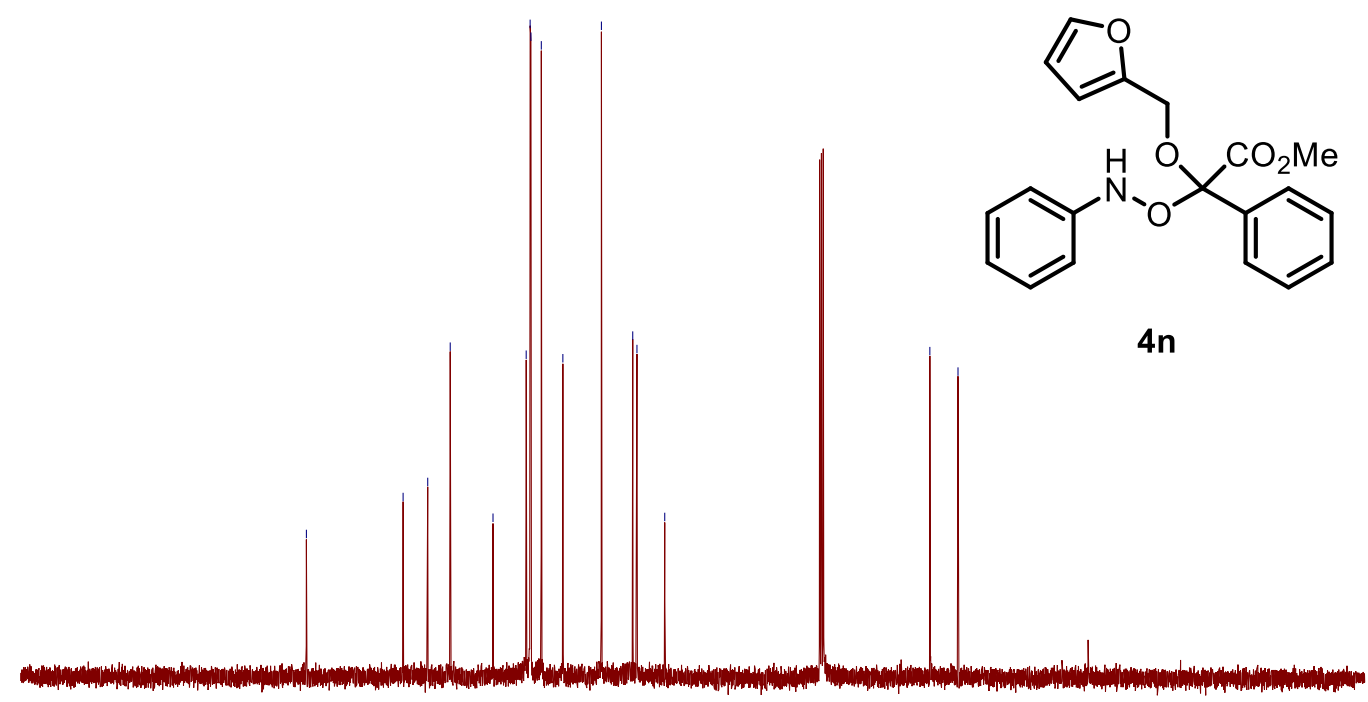

$21020019018017016015014013012011010090 \quad 80 \quad 70 \quad 60 \quad 50 \quad 40 \quad 30 \quad 20 \quad 10 \quad 0 \quad-10$

${ }^{13} \mathrm{C}\left\{{ }^{1} \mathrm{H}\right\} \mathrm{NMR}$ Spectrum of Compound $4 \mathbf{n}\left(100 \mathrm{MHz}, \mathrm{CDCl}_{3}\right)$.

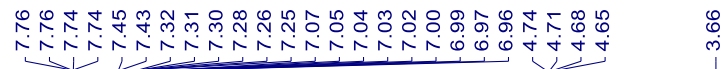

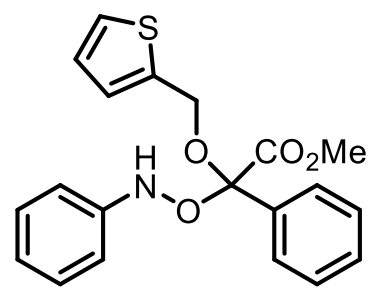

40

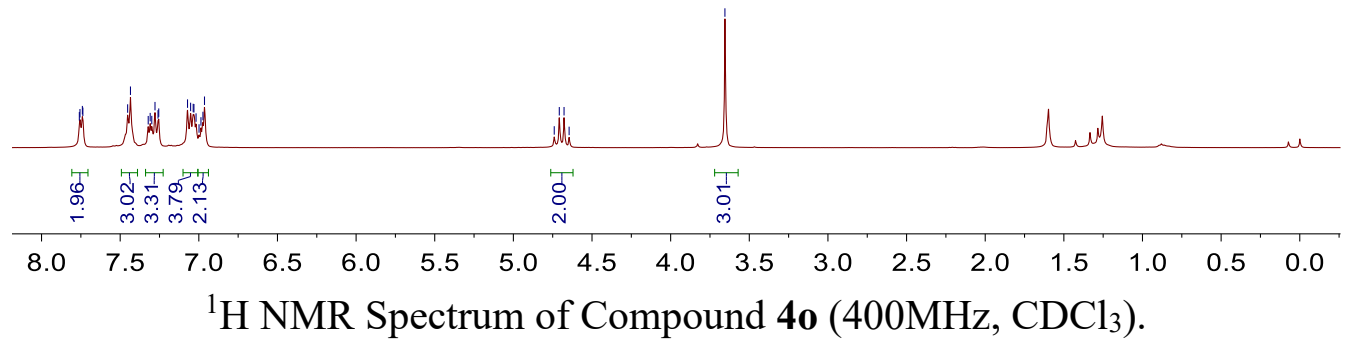




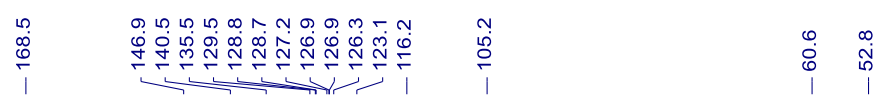

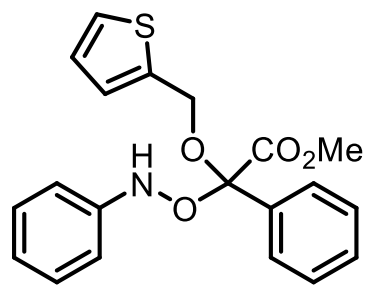

4o

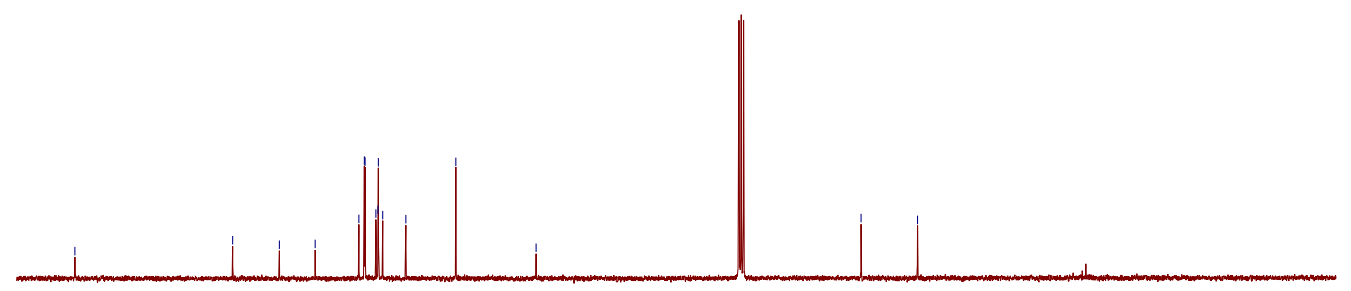

$\begin{array}{llllllllllllllllll}170 & 160 & 150 & 140 & 130 & 120 & 110 & 100 & 90 & 80 & 70 & 60 & 50 & 40 & 30 & 20 & 10 & 0\end{array}$

${ }^{13} \mathrm{C}\left\{{ }^{1} \mathrm{H}\right\} \mathrm{NMR}$ Spectrum of Compound $40\left(100 \mathrm{MHz}, \mathrm{CDCl}_{3}\right)$.

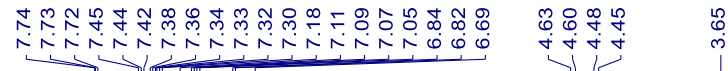

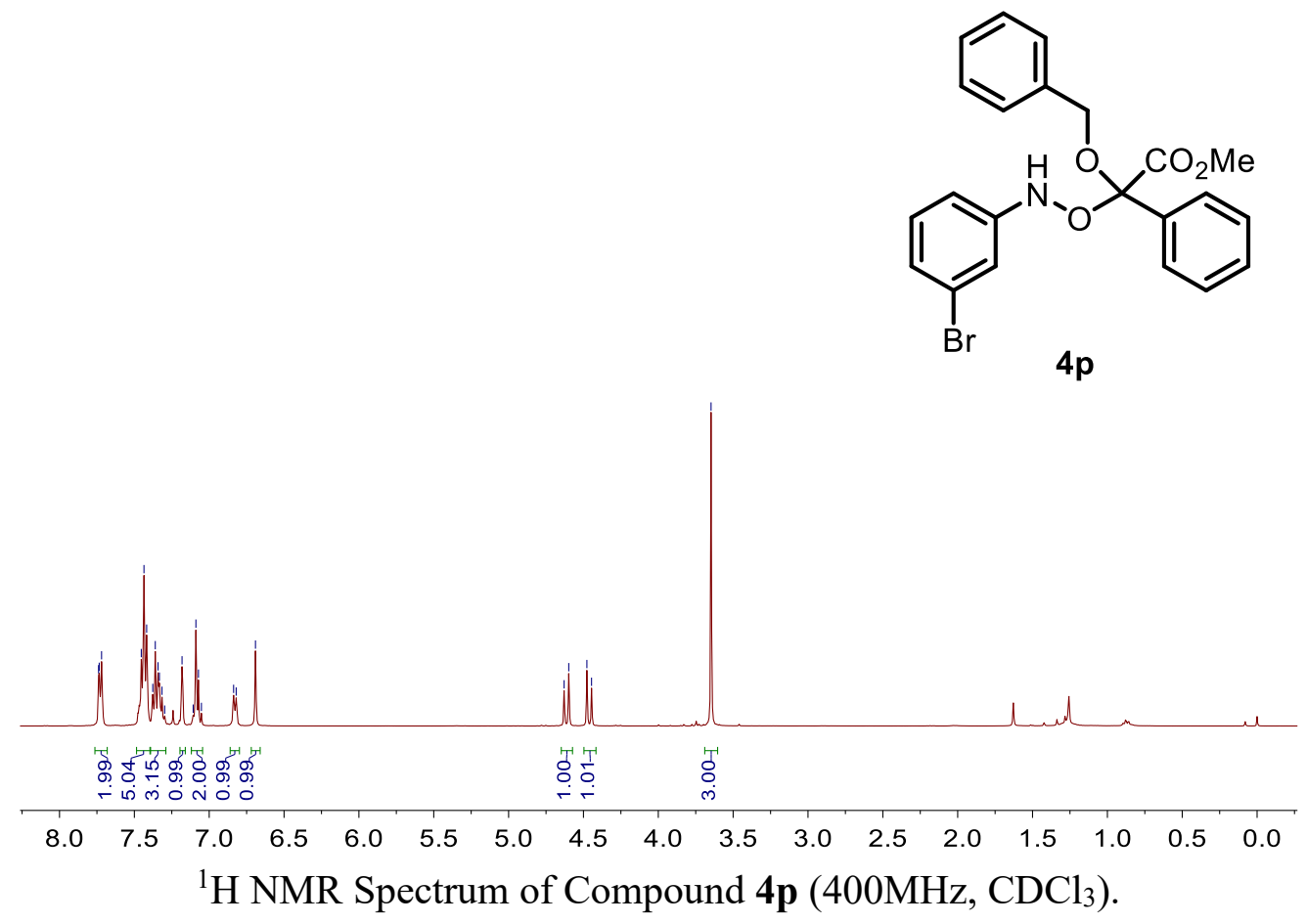




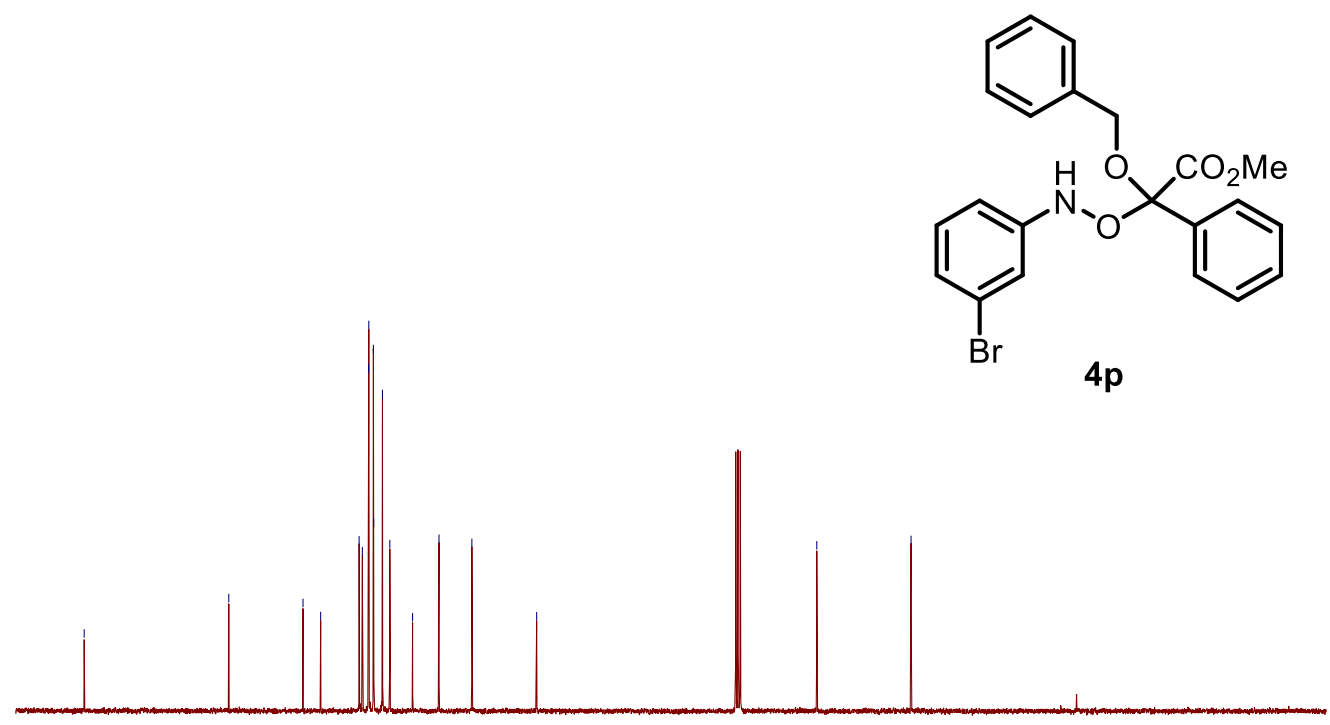

$\begin{array}{llllllllllllllllll}170 & 160 & 150 & 140 & 130 & 120 & 110 & 100 & 90 & 80 & 70 & 60 & 50 & 40 & 30 & 20 & 10 & 0\end{array}$

${ }^{13} \mathrm{C}\left\{{ }^{1} \mathrm{H}\right\} \mathrm{NMR}$ Spectrum of Compound $4 \mathbf{p}\left(100 \mathrm{MHz}, \mathrm{CDCl}_{3}\right)$.

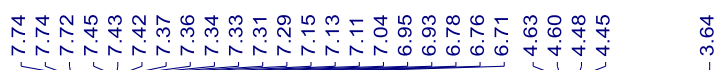

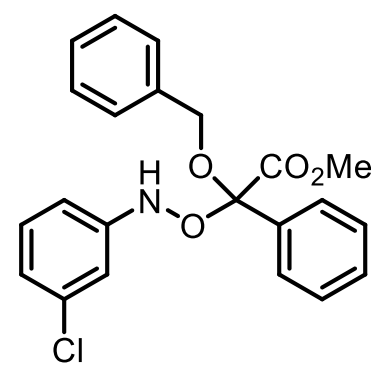

$4 q$

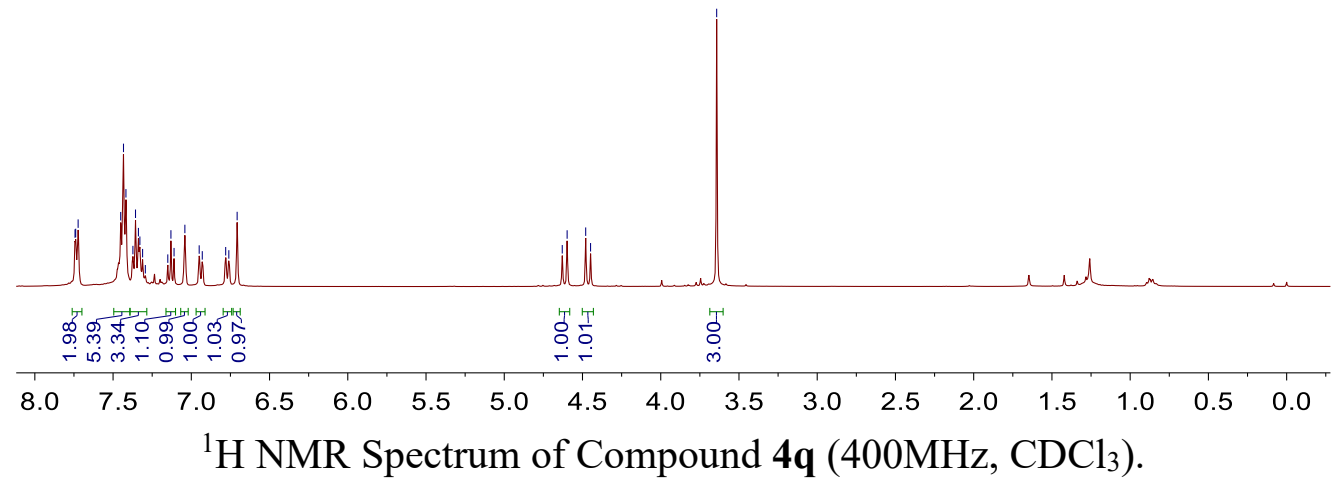




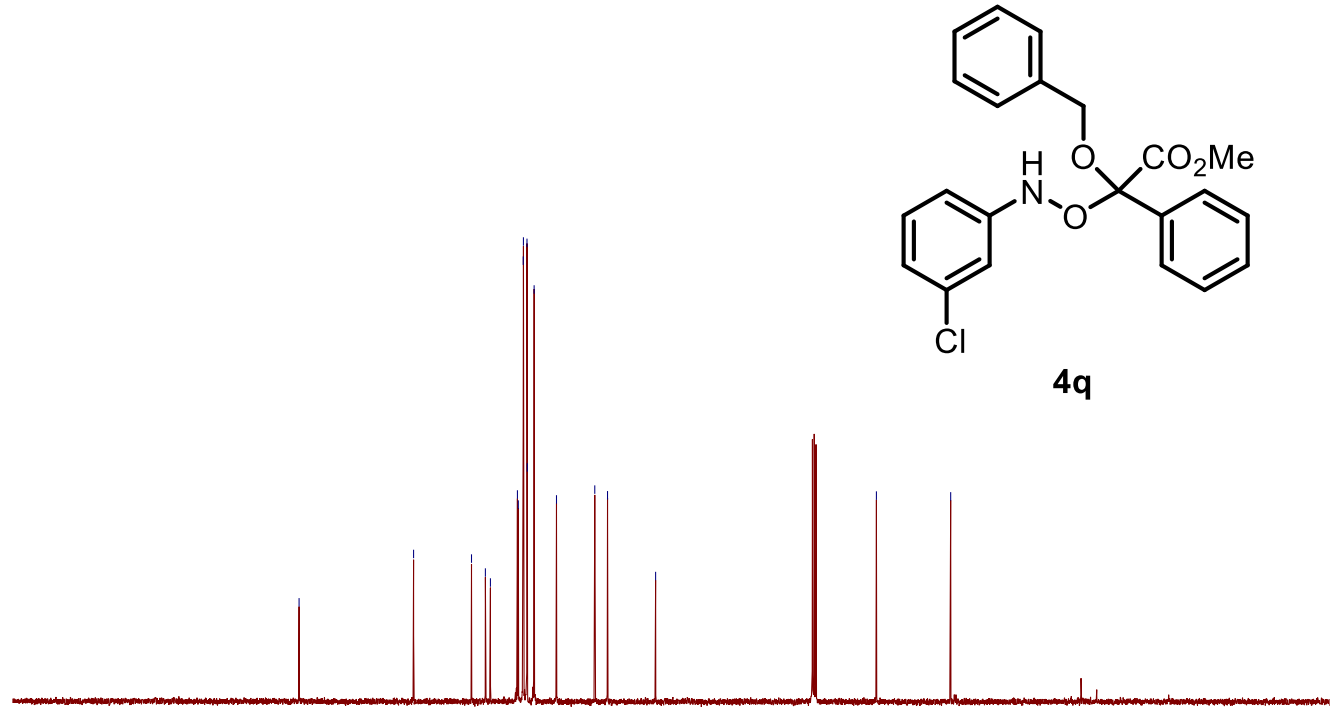

$210200190180170160150140130120110100 \quad 90 \quad 80 \quad 70 \quad 60 \quad 50 \quad 40 \quad 30 \quad 20 \quad 10 \quad 0 \quad-10$ ${ }^{13} \mathrm{C}\left\{{ }^{1} \mathrm{H}\right\} \mathrm{NMR}$ Spectrum of Compound $\mathbf{4 q}\left(100 \mathrm{MHz}, \mathrm{CDCl}_{3}\right)$. 